\title{
ALMA Observations of Starless Core Substructure in Ophiuchus
}

\author{
H. Kirk ${ }^{1}$, M. M. Dunham ${ }^{2,3}$, J. Di Francesco ${ }^{1,4}$, D. Johnstone ${ }^{1,4}$, S. S. R. Offner $^{5}$, S. I. Sadavoy ${ }^{3}$, J. J. Tobin ${ }^{6}$, H. G. Arce ${ }^{7}$, \\ $\mathrm{T}_{\mathrm{i}}$ L. Bourke $^{8}$, S. Mairs ${ }^{4}$, P. C. Myers ${ }^{3}$, J. E. Pineda ${ }^{9}$, S. Schnee ${ }^{10}$, and Y. L. Shirley ${ }^{11}$ \\ ${ }^{1}$ NRC Herzberg Astronomy and Astrophysics, 5071 West Saanich Road, Victoria, BC, V9E 2E7, Canada \\ ${ }^{2}$ Department of Physics, State University of New York at Fredonia, 280 Central Avenue, Fredonia, NY 14063, USA \\ ${ }^{3}$ Harvard-Smithsonian Center for Astrophysics, 60 Garden Street, MS 78, Cambridge, MA 02138, USA \\ ${ }^{4}$ Department of Physics and Astronomy, University of Victoria, Victoria, BC, V8P 1A1, Canada \\ ${ }^{5}$ Department of Astronomy, University of Massachusetts, Amherst, MA 01002, USA \\ ${ }^{6}$ Department of Physics and Astronomy, University of Oklahoma, 440 W. Brooks Street, 231 Nielsen Hall, \\ Norman, OK 73019, USA \\ ${ }^{7}$ Department of Astronomy, Yale University, P.O. Box 208101, New Haven, CT 06520, USA \\ ${ }^{8}$ SKA Organization, Jodrell Bank Observatory, Lower Withington, Macclesfield, Cheshire SK11 9DL, UK \\ ${ }_{9}$ Max-Planck-Institut fr extraterrestrische Physik, Giessenbachstrasse 1, D-85748, Garching, Germany \\ ${ }^{10}$ National Radio Astronomy Observatory, 520 Edgemont Road, Charlottesville, VA 22903, USA \\ ${ }^{11}$ Steward Observatory, University of Arizona, 933 North Cherry Avenue, Tucson, AZ 85721, USA \\ Received 2016 November 27; revised 2017 February 21; accepted 2017 February 27; published 2017 March 31
}

\begin{abstract}
Compact substructure is expected to arise in a starless core as mass becomes concentrated in the central region likely to form a protostar. Additionally, multiple peaks may form if fragmentation occurs. We present Atacama Large Millimeter/submillimeter Array (ALMA) Cycle 2 observations of 60 starless and protostellar cores in the Ophiuchus molecular cloud. We detect eight compact substructures which are $>15^{\prime \prime}$ from the nearest Spitzer young stellar object. Only one of these has strong evidence for being truly starless after considering ancillary data, e.g., from Herschel and X-ray telescopes. An additional extended emission structure has tentative evidence for starlessness. The number of our detections is consistent with estimates from a combination of synthetic observations of numerical simulations and analytical arguments. This result suggests that a similar ALMA study in the Chamaeleon I cloud, which detected no compact substructure in starless cores, may be due to the peculiar evolutionary state of cores in that cloud.
\end{abstract}

Key words: Stars: formation - submillimeter: ISM

\section{Introduction}

Protostars form within dense cores, which in turn are usually found embedded within larger molecular cloud complexes. Furthermore, many stars are found in binary or higher-order multiple systems (roughly half of solar-type stars, e.g., Duquennoy \& Mayor 1991; Raghavan et al. 2010; Duchêne \& Kraus 2013), and there is strong evidence to suggest that multiplicity may be more common in younger protostellar systems (e.g., Looney et al. 2000; Connelley et al. 2008; Chen et al. 2013; Tobin et al. 2016b). A high protostellar multiplicity is also expected based on models comparing the core mass function (CMF) and initial stellar mass function (Goodwin et al. 2008; Holman et al. 2013; Lomax et al. 2015). While the formation of binary and higher-order protostellar systems could arise through fragmentation of a protostellar disk (e.g., Kratter \& Lodato 2016; Tobin et al. 2016a), some of these multiple systems could also begin forming earlier, at the starless core stage. If so, then dense cores likely have a more complex structure than the traditional picture of a radially symmetric, smooth density profile which is flat in the center and decreases as a roughly $r^{-2}$ power law on the outskirts (e.g., Di Francesco et al. 2007).

One mechanism for generating anisotropic structure in cores is turbulence. In the model of turbulent fragmentation, for example, turbulence creates denser sub-regions within a starless core. These denser sub-regions can then individually become Jeans unstable and collapse, later forming multiple systems with larger (thousands of au) separations (Fisher 2004; Goodwin et al. 2004; Offner et al. 2010, 2016). Interferometers, especially the Atacama Large Millimeter/submillimeter Array
(ALMA), should be sensitive to substructures generated by processes such as turbulent fragmentation for at least the later stages of a starless core's evolution, just before the protostar forms (Offner et al. 2012; Mairs et al. 2014; Dunham et al. 2016).

The detection rate of such compact substructures in starless cores yields clues to the processes driving their formation. For example, Dunham et al. (2016) find that for an ALMA observing set-up mimicking their observations of starless cores in Chamaeleon I (discussed in more detail below), compact structures generated in starless cores by turbulence should have a detection rate more than 10 times higher than comparable detections of simple smooth Bonnor-Ebert sphere-like (Ebert 1955; Bonnor 1956) cores. This difference is attributable to the much smoother density profile of the Bonnor-Ebert sphere than the structures generated in numerical simulations. It is only at very high central densities that there is sufficient variation in the small-scale density structure of the Bonnor-Ebert sphere to be visible after interferometry filters out the larger-scale emission structures.

The search for compact substructure within starless cores with interferometers has typically targeted sources with signs of being near protostellar collapse (e.g., Kirk et al. 2009; Chen \& Arce 2010; Pineda et al. 2011, 2015; Bourke et al. 2012; Nakamura et al. 2012; Lee et al. 2013; Friesen et al. 2014; Kainulainen et al. 2016). Many of these studies focused on isolated Bok globules rather than on starless cores within larger-scale clouds, and all but two of those have small samples of one or two sources. The two exceptions are Lee et al. (2013), who surveyed eight cores but only detected compact 
substructure in molecular line emission and not continuum emission, and Kainulainen et al. (2016), discussed in more detail in Section 5.2. Larger continuum surveys of many starless cores in the same cloud tend to yield a low detection rate. Schnee et al. (2010) initially reported two detections in a sample of 11 starless cores in Perseus, but both of these objects were later shown to be very low-luminosity protostars (Schnee et al. 2012). These non-detections imply that most of the starless cores surveyed have smooth, extended structure which would be filtered out by interferometric observations.

Before the advent of ALMA and the Jansky Very Large Array (JVLA), interferometric observations of dense cores required a significant investment of telescope time per core, therefore limiting the above-mentioned studies to relatively small numbers of targets, with limited sensitivity to substructure on a range of scales. Using synthetic observations of numerical simulations of star formation under the turbulent fragmentation scenario, Offner et al. (2012) demonstrated that ALMA should have the sensitivity to detect core substructure, especially when the full array is available and the observations include data from the ALMA Compact Array that trace slightly larger angular scales. The JVLA is similarly being used as an effective probe of protostellar multiplicity (Tobin et al. 2016b). ALMA and JVLA observations additionally require significantly less integration time to reach the same sensitivity as earlier interferometric arrays, making larger population studies more easily achievable.

To date, the only ALMA survey of starless cores was published by Dunham et al. (2016), hereafter D16. They presented ALMA Cycle 1 observations of the entire population of dense cores in the Chamaeleon I cloud, 73 sources in total, at a distance of $150 \mathrm{pc}$, of which none of the 56 starless cores were detected. Based on simulations of turbulent cores, discussed in more detail in Section 5, D16 expected at least two detections of compact substructure in starless cores. To reconcile the lack of detections with the expected number, D16 argued that Chamaeleon I may not be continuously forming stars. If all of the starless cores in Chamaeleon I are at an early evolutionary phase or will dissipate rather than collapse to form protostars, then it is unlikely any substructure is sufficiently well-developed to be observable there. Alternatively, if the starless cores in Chamaeleon I can be well-described by a simple, smooth Bonnor-Ebert sphere model, no detections would be expected. Observations of starless core populations in additional molecular clouds are therefore crucial to determine whether a "typical" population of dense cores has structures that are more complex than the simple Bonnor-Ebert sphere model.

The Ophiuchus molecular cloud presents an optimal environment to extend the D16 study. Ophiuchus is one of the nearest molecular clouds, at only $\sim 140 \mathrm{pc}$ (Ortiz-León et al. 2017), similar to Chamaeleon. Its dense core population has been characterized in the (sub)millimeter continuum by a variety of authors (Motte et al. 1998; Johnstone et al. 2000, 2004; Nutter et al. 2006; Stanke et al. 2006; Young et al. 2006; Enoch et al. 2008; Jørgensen et al. 2008; Pattle et al. 2015), and its young stellar object (YSO) population has been characterized with Spitzer (Dunham et al. 2008; Jørgensen et al. 2008; Padgett et al. 2008; Sadavoy et al. 2010a).

Here, we present ALMA Cycle 2 observations of the dense cores in Ophiuchus to search for signs of compact substructure.
We note that the angular resolution of our observations would allow us to see multiple peaks generated under a turbulent fragmentation type of model (at thousands of au scales), in addition to compact peaks, which still allow us to test for the action of processes beyond the simple evolution of a BonnorEbert sphere. Our resolution is insufficient, however, to reveal small separation multiples (at hundreds of au), which might form via disk fragmentation.

We describe our observations and existing applicable data in Section 2, and present the ALMA detections and their basic properties in Section 3. In Section 4, we compare the properties of candidate starless detections with their protostellar counterparts. In Section 5, we interpret the candidate starless core detections in the context of the turbulent fragmentation model, and in Section 6, we summarize our conclusions.

\section{Observations}

\subsection{Target Selection: SCUBA}

In their joint analysis of SCUBA $850 \mu \mathrm{m}$ and multiwavelength Spitzer data, Jørgensen et al. (2008) identified 66 dense cores in the Ophiuchus molecular cloud. We apply a simple definition that protostellar dense cores are separated by no more than $15^{\prime \prime}$ (i.e., roughly one SCUBA $850 \mu \mathrm{m}$ beam) from one (or more) Spitzer-identified YSOs. Using this requirement, we conservatively classify 43 cores as starless and 23 cores as protostellar. We use the updated Spitzer YSO catalog of Dunham et al. (2015) for this determination, and further note that of the 23 protostellar cores, 14 are associated with a Class $0+\mathrm{I}$ YSO, four are associated with a Class "Flat" YSO, and five are associated with a Class II YSO. These classifications are based on the extinction-corrected slope of the spectral energy distribution measured across the Spitzer bands ( $\alpha_{0}$ from Dunham et al. 2015).

None of the 43 starless cores in our list contains candidate embedded YSOs in Jørgensen et al. (2008) or are included in the candidate faint embedded YSO list of Dunham et al. (2008). Two of the starless cores in our list (162628-24235 and 162628-24225), however, match cores that Sadavoy et al. (2010a) identify as having evidence of a protostellar nature.

We obtained ALMA observations for all 66 of the dense cores identified in Jørgensen et al. (2008). Figures 1 and 2 show the positions of the 66 pointing centers overlaid on the recent SCUBA-2 $850 \mu \mathrm{m}$ map of Ophiuchus from Pattle et al. (2015).

\subsubsection{Comparison of SCUBA and SCUBA-2 Data}

Although not available when the ALMA observations were proposed, the Ophiuchus cloud was recently observed and analyzed by the JCMT Gould Belt Survey by Pattle et al. (2015), which has larger spatial coverage and better flux sensitivity than the original SCUBA observations used to create the ALMA target list. We examined our adopted dense core catalog using this improved SCUBA-2 data set. Unfortunately, six of the cores identified by Jørgensen et al. appear to be spurious objects: 162614-24232, 162616-24235, 16264424253, 162646-24242, 163248-23523, and 163248-23524. All six appear to be part of larger structures which were identified as other sources in the Jørgensen et al. (2008) catalog. Examining the original SCUBA maps, it appears that a combination of poor larger-scale flux recovery and a higher local pixel-to-pixel noise caused the core identifying algorithm 


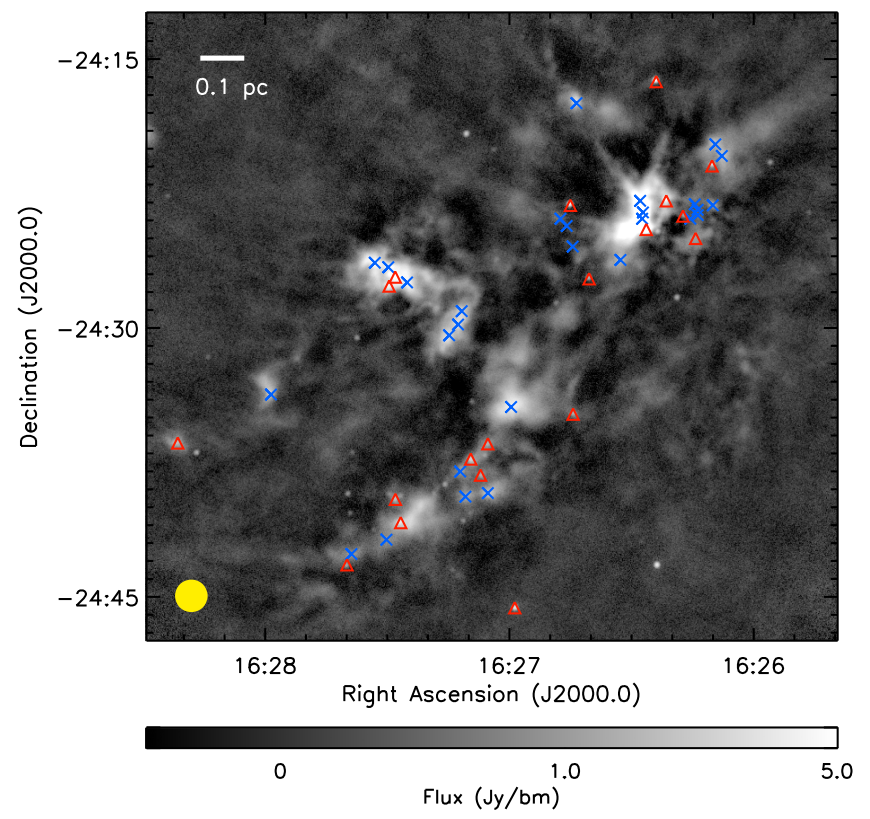

Figure 1. Location of the dense cores targeted in our ALMA observations in the western portion of Ophiuchus. The background grayscale image shows the SCUBA-2 $850 \mu \mathrm{m}$ map created using the JCMT GBS Data Release 2 reduction method (H. Kirk et. al. 2017, in preparation). The blue crosses show starless dense cores, while the red triangles show protostellar dense cores. The filled yellow circle at the bottom left corner indicates the size of the ALMA primary beam. The horizontal line in the upper left corner indicates a length of $0.1 \mathrm{pc}$ at the assumed distance to Ophiuchus of $140 \mathrm{pc}$.

they used in their analysis (clumpfind; Williams et al. 1994) to subdivide the emission artificially into multiple structures. (Challenges with using clumpfind are discussed in detail in Pineda et al. 2009.) Since all six sources are the result of artificial subdivision of larger emission structures which were also included in our catalog and observed, the ALMA observations serve to provide deeper integrations on those adjacent real dense cores. All six spurious sources fall into our starless core category, giving us a total of 37 starless cores and 23 protostellar cores. For the remainder of our analysis, we therefore consider our observational sample to include 60 cores, and use the data from the six spurious cores only to improve the sensitivity toward their neighboring real cores.

We furthermore compared the SCUBA-based core catalog with the core catalog presented in Pattle et al. (2015) using SCUBA-2 observations. Pattle et al. (2015) used the corefinding algorithm CuTEx (Molinari et al. 2011), which uses the second derivative of the emission map to identify objects. In practice, this approach renders CuTEx insensitive to larger, fluffier sources, while giving it an advantage in breaking up complex clustered emission into multiple sources. As such, we find that some of the fainter SCUBA cores are not listed in the Pattle et al. (2015) catalog, despite there being visually obvious emission at their locations. Moreover, other SCUBA cores are associated with multiple listings in the Pattle et al. (2015) catalog, as CuTEx subdivided the respective emission to a much greater extent than clumpfind did. We emphasize that, in general, there is good agreement between the two catalogs for the brightest cores, while fainter cores have more likelihood to differ for the reasons outlined above. Since there is not a oneto-one correspondence between the two catalogs, for the

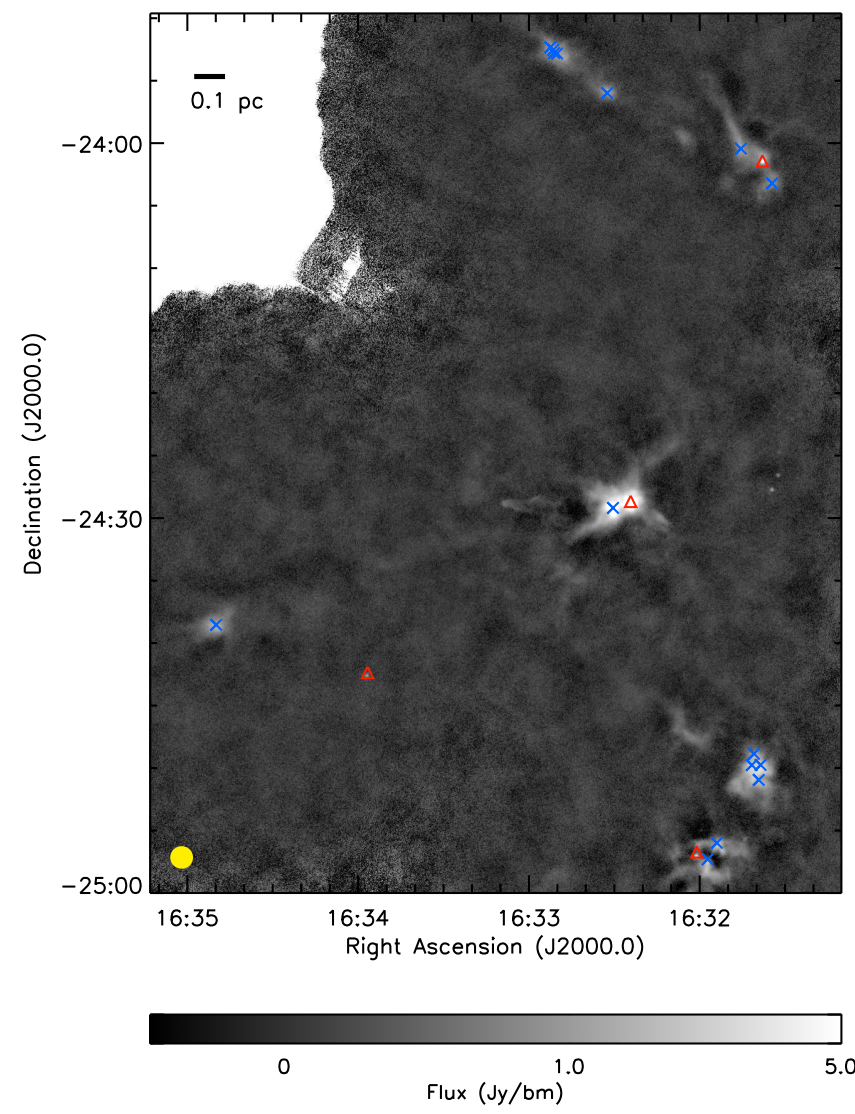

Figure 2. Location of the dense cores targeted in our ALMA observations in the eastern portion of Ophiuchus. The background grayscale image shows the SCUBA-2 $850 \mu \mathrm{m}$ map created using the JCMT GBS Data Release 2 reduction method (H. Kirk et al. 2017, in preparation). The blue crosses show starless dense cores, while the red triangles show protostellar dense cores. The filled yellow circle at the bottom left corner indicates the size of the ALMA primary beam. The horizontal line in the upper left corner indicates a length of $0.1 \mathrm{pc}$ at the assumed distance to Ophiuchus of $140 \mathrm{pc}$.

remainder of our analysis we present the properties derived for the cores from the SCUBA data in Jørgensen et al. (2008).

\subsection{ALMA Data}

The ALMA Band 3 data were observed during Cycle 2 on 2015 January 31 with 37 antennas. The array was in a relatively compact configuration, with a median synthesized beam of 3 . $5 \times 1$ ". 8 at a position angle of $71^{\circ}$, and a maximum recoverable scale of $\sim 23^{\prime \prime}$. The former corresponds to a physical size of $\sim 0.002 \mathrm{pc}$ or 350 au at a distance of $140 \mathrm{pc}$, while the latter corresponds to $\sim 0.016 \mathrm{pc}$. Our observational setup mimics that described in D16 for their earlier observations in Chamaeleon I. Only the $12 \mathrm{~m}$ array was used, i.e., no compact array or total power observations were included. Antenna separations ranged from $\sim 15$ to $350 \mathrm{~m}$. Each core was observed twice with a single pointing using roughly $1 \mathrm{~min}$ of on-source integration time. Three of the four spectral windows available were configured for continuum measurements at 101, 103, and $113 \mathrm{GHz}$, each covering a bandwidth of $2 \mathrm{GHz}$, and giving an effective mean frequency of $107 \mathrm{GHz}$. The noise level of the continuum observations about the phase centers is roughly $0.16 \mathrm{mJy}$ beam $^{-1}$. The remaining spectral window 
was configured to observe ${ }^{12} \mathrm{CO}(1-0)$ emission at $115 \mathrm{GHz}$; the line data are not presented here.

We used the pipeline calibrated data available for Cycle 2 data (Shinnaga et al. 2015). For fields where a bright enough source was detected, we manually self-calibrated the data in both phase and amplitude using the Common Astronomy Software Applications (CASA) package, and we then imaged the data using manually defined clean boxes in an interactive clean session. In nearly all cases, self-calibration gave only a modest (ten percent or less) improvement on the rms noise level in the final image. Some of the observed cores lie sufficiently close together for their fields of view to overlap. In these cases, we mosaicked the fields together to improve the final sensitivity. Our observation of core 162628-24235 was omitted from our analysis, as it suffered from a known bug at ALMA, and gave inconsistent results. The area of core 16262824235 was covered by our ALMA observations of neighboring cores in the mosaic 162628-24225, so the exclusion of this one observation does not impact our science goals. Table 1 gives the final noise level for each core observed, along with our classification as starless or protostellar. Those cores lying within a mosaicked area are noted.

\section{Detections}

After correcting for the primary beam, we use CASA's imfit package to fit two-dimensional Gaussians to all compact sources of emission within the single maps and mosaicked areas. Table 2 lists the the 38 sources detected with ALMA and the association of each with the nearest SCUBA core (and separation), and nearest Spitzer YSO (and separation). Sources which do not have a close positional association with a Spitzer YSO, but have other information suggesting a protostellar nature (see Section 3.3) are also noted.

The results of the Gaussian fits to each ALMA detection are given in Table 3. Where possible, we calculate the beamdeconvolved size of the source. Marginally resolved sources are noted in Table 3 with upper limit signs for the deconvolved sizes and no position angles. Unresolved sources have no value listed for either a deconvolved size or position angle.

\subsection{Mass Estimates}

We can estimate the mass of each detected ALMA source using the standard equation

$$
M=100 \frac{d^{2} S_{\nu}}{B_{\nu}\left(T_{D}\right) \kappa_{\nu}}
$$

where $d$ is the distance, $S_{\nu}$ is the flux at frequency $\nu, B_{\nu}$ is the Planck function at a dust temperature of $T_{D}, \kappa_{\nu}$ is the dust opacity, and the factor of 100 represents the assumed gas-todust ratio. Following D16, we assume $\kappa_{\nu}=0.23 \mathrm{~cm}^{2} \mathrm{~g}^{-1}$ at an effective frequency of $107 \mathrm{GHz}$, which corresponds to the OH5 model of Ossenkopf \& Henning (1994). We assume a slightly warmer dust temperature for Ophiuchus than D16 assumed for Chamaeleon I (15 K versus $10 \mathrm{~K}$ ), based on the $\mathrm{NH}_{3}$ results of Friesen et al. (2009), and a distance of $140 \mathrm{pc}$. Note that on the small scales of the densest gas traced by ALMA, the temperatures of starless cores (i.e., source 37 and the extended emission discussed in Sections 3.4 and 3.5) are likely closer to a temperature of $10 \mathrm{~K}$, as discussed in Section 5. A temperature of $10 \mathrm{~K}$ would imply masses that are a factor of two higher than what we report. Conversely, the protostellar cores may be warmer on small size scales. A temperature of $25 \mathrm{~K}$ would imply true masses that are roughly a factor of two lower than what we report. The temperature and dust opacity contribute a factor of several to the uncertainty in the masses we estimate, while uncertainty in the distance has a smaller contribution.

Table 4 summarizes the estimated mass of each source detected with ALMA, in addition to their effective radius and mean density. We calculate the effective radius as the geometric mean of the deconvolved semimajor and semiminor axes (i.e., half of the reported FWHM values reported in Table 3). In the case of unresolved sources, we use the (clean) beam major and minor axes as an upper limit. Following D16, we calculate the mean density of each source as

$$
n=\frac{3}{4 \pi \mu m_{\mathrm{H}}} \frac{M}{R_{\mathrm{eff}}^{3}}
$$

where $\mu=2.37$ is the mean molecular weight per particle and $m_{\mathrm{H}}$ is the mass of a hydrogen atom.

For all quantities reported in Table 4, the uncertainty is dominated by systematic effects (distance, temperature, dust opacity, and source geometry), rather than the statistical uncertainties associated with each fit.

\subsection{Detections and Protostellar Associations}

In Table 2, we show the angular separation from each ALMA detection to the nearest Spitzer YSO in the catalog of Dunham et al. (2015). Figure 3 shows these angular separations, which appear to divide into three different regimes: very small separations $\left(<2^{\prime \prime}\right.$ between the ALMA detection and a Spitzer YSO), moderate separations $\left(\sim 4^{\prime \prime}-7^{\prime \prime}\right)$, and larger separations $\left(>14^{\prime \prime}\right)$. Some of our ALMA detections also lie significantly separated from the closest SCUBA core peak, as shown by the red filled histogram.

If the Spitzer catalog of Dunham et al. (2015) were complete, we would expect that all of our ALMA detections separated by more than a few arcsec from the nearest Spitzer YSO would be starless. (We interpret separations of $<2^{\prime \prime}$ as implying completely coincident ALMA and Spitzer sources, with the non-zero separation caused by pointing errors, etc; this is comparable to Spitzer's best angular resolution.) The presence of extended $24 \mu \mathrm{m}$ flux and saturated sources in portions of Ophiuchus, however, likely impacts the completeness of the Spitzer YSO catalog. As discussed in D16, there are several reasons to expect that even a complete Spitzer protostellar catalog does not capture the entire YSO population. For example, the Spitzer c2d program (Evans et al. 2009) missed identifying low-luminosity protostars due to insufficient sensitivity (Dunham et al. 2008), and these missing protostars will affect the classification of their host dense cores. Indeed, Schnee et al. (2010) detected two protostars with CARMA in their sample of 11 apparently starless cores in Perseus, both of which were too faint to have been detected by Spitzer c2d. Stutz et al. (2013) also find that some Orion protostars above a luminosity of $\sim 1 L_{\odot}$ are too faint at wavelengths of $24 \mu \mathrm{m}$ and shorter to be identifiable in Spitzer's Orion maps. Herschel, 
Table 1

Noise Levels of ALMA Observations

\begin{tabular}{|c|c|c|c|c|}
\hline Field $^{\mathrm{a}}$ & $\mathrm{rms}\left(\mathrm{mJy} \text { beam }^{-1}\right)^{\mathrm{b}}$ & Mosaic Field? ${ }^{\mathrm{c}}$ & Protostellar? $^{\mathrm{d}}$ & Concentration $^{\mathrm{e}}$ \\
\hline $162608-24202$ & 0.155 & $162610-24206$ & $S$ & 0.34 \\
\hline $162610-24195$ & 0.155 & $162610-24206$ & $\mathrm{~S}$ & 0.34 \\
\hline $162610-24231$ & 0.164 & $\cdots$ & $S$ & 0.40 \\
\hline $162610-24206$ & 0.155 & $162610-24206$ & II & 0.65 \\
\hline $162614-24232^{*}$ & 0.129 & $162617-24235$ & S & 0.15 \\
\hline $162614-24234$ & 0.129 & $162617-24235$ & $S$ & 0.22 \\
\hline $162614-24250$ & 0.165 & $\cdots$ & $0+\mathrm{I}$ & 0.34 \\
\hline $162615-24231$ & 0.129 & $162617-24235$ & S & 0.17 \\
\hline $162616-24235^{*}$ & 0.129 & $162617-24235$ & $S$ & 0.20 \\
\hline $162617-24235$ & 0.129 & $162617-24235$ & $0+\mathrm{I}$ & 0.32 \\
\hline $162622-24225$ & 0.159 & $\ldots$ & $0+\mathrm{I}$ & 0.58 \\
\hline $162624-24162$ & 0.172 & $\cdots$ & II & 0.41 \\
\hline $162626-24243$ & 0.167 & $162628-24225$ & $0+\mathrm{I}$ & 0.80 \\
\hline $162627-24233$ & 0.167 & $162628-24225$ & $S$ & 0.66 \\
\hline $162628-24235$ & 0.167 & $162628-24225$ & $S$ & 0.80 \\
\hline $162628-24225$ & 0.167 & $162628-24225$ & S & 0.66 \\
\hline $162633-24261$ & 0.162 & $\ldots$ & S & 0.41 \\
\hline $162641-24272$ & 0.156 & $\ldots$ & $0+\mathrm{I}$ & 0.53 \\
\hline $162644-24173$ & 0.166 & $\cdots$ & S & 0.40 \\
\hline $162644-24345$ & 0.170 & $\ldots$ & $0+\mathrm{I}$ & 0.29 \\
\hline $162644-24253^{*}$ & 0.168 & $\ldots$ & S & 0.23 \\
\hline $162645-24231$ & 0.159 & $\cdots$ & II & 0.74 \\
\hline $162646-24242^{*}$ & 0.114 & $162646-24242$ & $\mathrm{~S}$ & 0.16 \\
\hline $162648-24236$ & 0.114 & $162646-24242$ & $\mathrm{~S}$ & 0.33 \\
\hline $162659-24454$ & 0.163 & $\ldots$ & II & 0.42 \\
\hline $162660-24343$ & 0.164 & $\ldots$ & $\mathrm{S}$ & 0.55 \\
\hline $162705-24363$ & 0.155 & $\ldots$ & $0+\mathrm{I}$ & 0.34 \\
\hline $162705-24391$ & 0.168 & $\cdots$ & S & 0.44 \\
\hline $162707-24381$ & 0.171 & $162709-24372$ & $0+\mathrm{I}$ & 0.22 \\
\hline $162709-24372$ & 0.171 & $162709-24372$ & $0+\mathrm{I}$ & 0.48 \\
\hline $162711-24393$ & 0.160 & $\ldots$ & S & 0.29 \\
\hline $162712-24290$ & 0.164 & $\cdots$ & $\mathrm{S}$ & 0.21 \\
\hline $162712-24380$ & 0.145 & $\cdots$ & S & 0.31 \\
\hline $162713-24295$ & 0.158 & $\cdots$ & S & 0.38 \\
\hline $162715-24303$ & 0.157 & $\cdots$ & S & 0.34 \\
\hline $162725-24273$ & 0.146 & $162730-24264$ & $\mathrm{~S}$ & 0.37 \\
\hline $162727-24405$ & 0.158 & $\ldots$ & $0+\mathrm{I}$ & 0.57 \\
\hline $162728-24271$ & 0.146 & $162730-24264$ & Flat & 0.34 \\
\hline $162728-24393$ & 0.161 & $\ldots$ & $0+\mathrm{I}$ & 0.37 \\
\hline $162729-24274$ & 0.146 & $162730-24264$ & Flat & 0.50 \\
\hline $162730-24264$ & 0.146 & $162730-24264$ & S & 0.35 \\
\hline $162730-24415$ & 0.158 & $\ldots$ & S & 0.32 \\
\hline $162733-24262$ & 0.164 & $\ldots$ & $\mathrm{S}$ & 0.45 \\
\hline $162739-24424$ & 0.150 & $162740-24431$ & $S$ & 0.31 \\
\hline $162740-24431$ & 0.150 & $162740-24431$ & Flat & 0.27 \\
\hline $162759-24334$ & 0.141 & $\ldots$ & S & 0.46 \\
\hline $162821-24362$ & 0.155 & $\ldots$ & $0+\mathrm{I}$ & 0.39 \\
\hline $163133-24032$ & 0.165 & $\ldots$ & S & 0.35 \\
\hline $163136-24013$ & 0.149 & $\ldots$ & Flat & 0.72 \\
\hline $163138-24495$ & 0.152 & $\ldots$ & S & 0.38 \\
\hline $163139-24506$ & 0.157 & $\ldots$ & S & 0.35 \\
\hline $163140-24485$ & 0.163 & $\ldots$ & S & 0.29 \\
\hline $163141-24495$ & 0.163 & $\ldots$ & S & 0.36 \\
\hline $163143-24003$ & 0.164 & $\ldots$ & S & 0.33 \\
\hline $163154-24560$ & 0.172 & $\ldots$ & $\mathrm{S}$ & 0.40 \\
\hline $163157-24572$ & 0.159 & $\cdots$ & S & 0.46 \\
\hline $163201-24564$ & 0.155 & $\ldots$ & $0+\mathrm{I}$ & 0.45 \\
\hline $163223-24284$ & 0.827 & $\cdots$ & $0+\mathrm{I}$ & 0.94 \\
\hline $163229-24291$ & 0.167 & $\cdots$ & S & 0.74 \\
\hline $163230-23556$ & 0.166 & $\cdots$ & S & 0.33 \\
\hline $163247-23524$ & 0.097 & $163249-23521$ & S & 0.26 \\
\hline $163248-23524^{*}$ & 0.097 & $163249-23521$ & $\mathrm{~S}$ & 0.30 \\
\hline $163248-23523^{*}$ & 0.097 & $163249-23521$ & $\mathrm{~S}$ & 0.19 \\
\hline 163249-23521 & 0.097 & $163249-23521$ & $\mathrm{~S}$ & 0.28 \\
\hline
\end{tabular}


Table 1

(Continued)

\begin{tabular}{lcccc}
\hline \hline Field $^{\mathrm{a}}$ & ${\text { rms }\left(\mathrm{mJy} \mathrm{beam}^{-1}\right)^{\mathrm{b}}}$ & Mosaic Field? $^{\mathrm{c}}$ & Protostellar? $^{\mathrm{d}}$ & Concentration $^{\mathrm{e}}$ \\
\hline $163356-24420$ & 0.177 & $\ldots$ & II & 0.33 \\
$163448-24381$ & 0.147 & $\cdots$ & S & 0.38 \\
\hline
\end{tabular}

Notes.

${ }^{a}$ Observed SCUBA core name from Jørgensen et al. (2008). Asterisks denote the six starless cores from that sample which more sensitive SCUBA-2 observations show were likely false detections. See the text for more details.

b Typical map/mosaic rms value for areas with no detections calculated using CASA's imstat command. The rms was calculated on the non-primary-beam corrected image, ensuring that representative noise values could be accurately measured even for images where there was a bright central point source.

${ }^{\mathrm{c}}$ For areas with multiple SCUBA cores having contiguous ALMA coverage, the easternmost core in the covered zone is listed. Dashes denote isolated fields.

d Protostellar class based on the Spitzer catalog of Dunham et al. (2015). "S" denotes starless cores.

${ }^{\mathrm{e}}$ Central concentration of each core as reported in Jørgensen et al. (2008).

however, is able to detect them clearly at longer wavelengths. We searched through the literature to look for any evidence that the ALMA detections separated by more than $3^{\prime \prime}$ from a Dunham et al. (2015) Spitzer YSO may harbour hidden protostars.

\subsection{Unidentified Low-luminosity Protostars}

\subsubsection{Herschel}

We first searched for signs of compact protostellar emission in Herschel $70 \mu \mathrm{m}$ and $100 \mu \mathrm{m}$ observations of Ophiuchus, taken as part of the Herschel Gould Belt Survey (André et al. 2010), where the angular resolution is $\sim 5^{\prime \prime}$. Most of our ALMA detections not associated with a Spitzer YSO appear to have compact detections in the Herschel data, indicative of protostars. The one clear exception is ALMA source 37 which shows no signs of a protostellar association. A further three sources, ALMA sources 10, 11, and 12 are found in an area with complex, extended emission in the Herschel bands, making it difficult to ascertain the protostellar or starless nature of the individual detections. All four sources without clear protostellar counterparts in Herschel have Spitzer separations of $>14^{\prime \prime}$ in Figure 3.

\subsubsection{Published catalogs}

We also examined the full, uncut Spitzer c2d catalog ${ }^{12}$ to search for point sources which were excluded from the final YSO catalog due to confusion from extended emission or other selection criteria. Most of the Herschel protostar detections are also listed in the full Spitzer c2d catalog, with a range of classifications, highlighting the challenges of complete YSO identification in Ophiuchus. Of special note is that ALMA source 12 has a close positional match with Spitzer source $\mathrm{J} 162630.4-242257$, listed as a candidate YSO. This source is not present in the YSO candidate catalog of Evans et al. (2009), implying it failed to satisfy one or more additional criteria used to create the final catalog. Given the positional match with ALMA source 12, we consider this Spitzer detection to be a likely YSO.

We furthermore searched for associations between the the eight ALMA detections located more than 14" from Spitzer YSOs and YSO indicators in the literature via the SIMBAD database and other published catalogs.

\footnotetext{
${ }^{12}$ Available at http://irsa.ipac.caltech.edu/data/SPITZER/C2D/.
}

In SIMBAD (Wenger et al. 2000), we only found three cases of strong evidence for positional coincidence with a YSO. Three of the eight ALMA detections separated by more than 14" from a Spitzer YSO are located within 0." 6 of an $\mathrm{X}$-ray or joint X-ray and radio detection, and are likely to be YSOs. ALMA sources 10 and 11 both appear in Chandra (resolution 0 ".5) and VLA (resolution $\geqslant 2$ !"6) observations obtained by Gagné et al. (2004) and as such are very strong YSO candidates. Source 23 was detected by XMM-Newton (resolution $6^{\prime \prime}$ ) and classified as a candidate star (but not a pre-main sequence star) by Lin et al. (2012). There is also a T Tau type star located 0." 4 from this ALMA detection, which perhaps is tied to the X-ray detection. Sources 5, 12, 27, and 37 have only tentative associations with early infrared-based catalog objects which, if representing truly young protostellar sources, we would expect would be easily detectable with Spitzer. Source 19 has no match within $14^{\prime \prime}$ listed in SIMBAD.

None of the eight ALMA detections is listed as a source of molecular hydrogen outflows identified in Zhang et al. (2013).

Table 2 summarizes all of the strong lines of evidence of a protostellar association (i.e., Herschel, Spitzer, and X-ray plus radio catalogs) for each ALMA detection.

\subsubsection{ALMA CO}

If a young and faint protostar is associated with any of the ALMA detections, we might also see evidence of a protostellar outflow in our ALMA CO data. We defer a full analysis of the $\mathrm{CO}$ data for future work, but discuss here results from a quick check of the CO data for the eight ALMA detections located far from Spitzer YSOs. We ran a simple automated version of clean on the $\mathrm{CO}$ observations of the appropriate fields. We see no evidence for outflows around any of the positions of the eight ALMA detections. This lack of detection may not provide a strong constraint on the absence of a protostar, however, as without compact array or total power observations, it may be difficult to identify outflow emision.

\subsubsection{Summary}

Combining the results from all of these searches we find evidence that all of the ALMA detections separated by $4^{\prime \prime}$ to $7^{\prime \prime}$ to the nearest Dunham et al. (2015) Spitzer YSO are protostellar. Nearly all of the eight ALMA detections separated by at least $14^{\prime \prime}$ from the nearest Spitzer YSO are also 
Table 2

ALMA Detections and Associations

\begin{tabular}{|c|c|c|c|c|c|c|c|}
\hline $\begin{array}{l}\mathrm{Src}^{\mathrm{a}} \\
\#\end{array}$ & $\begin{array}{l}\text { R.A. }^{\mathrm{a}} \\
\text { (J2000) }\end{array}$ & $\begin{array}{l}\text { Decl. }^{\mathrm{a}} \\
\text { (J2000) }\end{array}$ & $\begin{array}{l}\text { SCUBA }^{b} \\
\text { Core }\end{array}$ & $\begin{array}{c}\text { Sep. } \\
\left(^{\prime \prime}\right)\end{array}$ & $\begin{array}{l}\text { Spitzer }^{\mathrm{c}} \\
\text { Match? }\end{array}$ & $\begin{array}{l}\text { Sep. }^{\mathrm{c}} \\
\left({ }^{\prime \prime}\right)\end{array}$ & $\begin{array}{l}\text { Common }^{\mathrm{d}} \\
\text { Name }\end{array}$ \\
\hline 1 & $16: 26: 10.33$ & $-24: 20: 55.17$ & $162610-24206$ & 2.6 & J162610.3-242054 & 1.2 & $\ldots$ \\
\hline 2 & $16: 26: 17.24$ & $-24: 23: 45.72$ & $162617-24235$ & 3.7 & J162617.2-242345 & 0.9 & $\ldots$ \\
\hline 4 & $16: 26: 21.39$ & $-24: 23: 04.78$ & $162622-24225$ & 10.4 & $\mathrm{~J} 162621.3-242304$ & 1.4 & $\ldots$ \\
\hline 5 & $16: 26: 21.73$ & $-24: 22: 50.93$ & $162622-24225$ & 4.3 & Hersch. & 14.3 & $\ldots$ \\
\hline 6 & $16: 26: 24.09$ & $-24: 16: 13.81$ & $162624-24162$ & 2.1 & J162624.0-241613 & 1.5 & $\ldots$ \\
\hline 9 & $16: 26: 26.36$ & $-24: 24: 30.72$ & $162626-24243$ & 1.9 & J162626.4-242430 & 0.9 & VLA 1623 \\
\hline 10 & $16: 26: 27.43$ & $-24: 24: 18.26$ & $162626-24243$ & 17.9 & X-ray + Hersch.? & 18.3 & $\ldots$ \\
\hline 11 & $16: 26: 27.86$ & $-24: 23: 59.56$ & $162628-24235$ & 6.7 & X-ray + Hersch.? & 33.1 & OphA-SM1 \\
\hline 12 & $16: 26: 30.48$ & $-24: 22: 57.55$ & $162628-24225$ & 34.0 & c2d-uncut + Hersch.? & 69.5 & $\ldots$ \\
\hline 13 & $16: 26: 40.47$ & $-24: 27: 14.90$ & $162641-24272$ & 1.3 & $\mathrm{~J} 162640.4-242714$ & 1.3 & $\ldots$ \\
\hline 14 & $16: 26: 44.21$ & $-24: 34: 48.81$ & $162644-24345$ & 2.7 & $\mathrm{~J} 162644.1-243448$ & 1.6 & $\ldots$ \\
\hline 19 & $16: 27: 05.86$ & $-24: 37: 08.36$ & $162705-24363$ & 40.7 & Hersch. & 40.4 & $\ldots$ \\
\hline 20 & $16: 27: 06.76$ & $-24: 38: 15.21$ & $162707-24381$ & 4.9 & $\mathrm{~J} 162706.7-243814$ & 1.5 & $\ldots$ \\
\hline 21 & $16: 27: 09.41$ & $-24: 37: 18.96$ & $162709-24372$ & 1.3 & J162709.4-243718 & 1.0 & $\ldots$ \\
\hline 22 & $16: 27: 09.49$ & $-24: 37: 22.89$ & $162709-24372$ & 3.5 & Hersch. & 5.0 & $\ldots$ \\
\hline 23 & $16: 27: 26.47$ & $-24: 39: 23.42$ & $162728-24393$ & 23.5 & X-ray + Hersch. & 21.6 & $\ldots$ \\
\hline 24 & $16: 27: 26.60$ & $-24: 40: 45.51$ & $162727-24405$ & 6.9 & Hersch. & 6.2 & $\ldots$ \\
\hline 25 & $16: 27: 26.91$ & $-24: 40: 50.71$ & $162727-24405$ & 3.4 & $\mathrm{~J} 162726.9-244050$ & 0.7 & $\ldots$ \\
\hline 26 & $16: 27: 28.00$ & $-24: 39: 33.76$ & $162728-24393$ & 0.5 & J162727.9-243933 & 1.6 & $\ldots$ \\
\hline 27 & $16: 27: 29.44$ & $-24: 39: 16.12$ & $162728-24393$ & 26.8 & Hersch. & 27.0 & $\ldots$ \\
\hline 28 & $16: 27: 30.18$ & $-24: 27: 43.74$ & $162729-24274$ & 9.9 & $\mathrm{~J} 162730.1-242743$ & 1.3 & $\ldots$ \\
\hline 29 & $16: 27: 37.22$ & $-24: 42: 38.51$ & $162739-24424$ & 21.6 & J162737.2-244237 & 1.5 & $\ldots$ \\
\hline 30 & $16: 27: 39.82$ & $-24: 43: 15.22$ & $162740-24431$ & 2.3 & J162739.8-244315 & 0.4 & $\ldots$ \\
\hline 31 & $16: 28: 21.63$ & $-24: 36: 23.78$ & $162821-24362$ & 3.2 & J162821.6-243623 & 0.9 & IRAS $16253-2429$ \\
\hline
\end{tabular}

Notes.

${ }^{\text {a }}$ Running index number and peak position of the ALMA detection.

${ }^{\mathrm{b}}$ Closest SCUBA core from Jørgensen et al. (2008), and the angular separation between the SCUBA core center and the ALMA detection's peak position.

${ }^{c}$ Protostellar associations. Angular separations to the nearest Spitzer YSO in Dunham et al. (2015) are given. The Dunham et al. (2015) source names are listed for sources with separations of less than $3^{\prime \prime}$. Associations with Herschel emission suggesting the presence of a protostar, an X-ray candidate YSO, or a YSO candidate in the original Spitzer c2d catalog are listed as appropriate for sources without a close Dunham et al. (2015) Spitzer match.

d Commonly known protostellar associations, where applicable.

protostellar. Sources 10, 11, 12, 17, 19, 23, and 27 all have evidence suggesting positional association with a protostar. Source 37 is a strong starless core candidate, and has a small (3")3) separation from the nearest SCUBA core peak, as expected for a starless core detection. These results are summarized in Table 2, which includes multiple lines of evidence of a protostellar nature where appropriate.

\subsection{Candidate Starless Core Detections}

Figure 4 shows the ALMA image for source 37, our best candidate starless core detection, which was detected at a level of $4.9 \sigma$. Sadavoy et al. (2010b) identified this starless core as one of only a handful in Ophiuchus, Taurus, Perseus, and Orion, that appear to be super-Jeans (having a mass of roughly twice the Jeans mass), and starless, and suggest it is a strong candidate for collapse. Lis et al. (2016) also identified a source coincident with our detection using a combination of ALMA Compact Array and CSO observations, and list it as the starless core L1689N.

\subsection{Extended Emission}

In addition to the compact sources, two of the mosaics show signs of extended emission. The more obvious of these is shown in Figure 5, where the dense ridge around Oph-SM1 
Table 3

Observed Properties of ALMA Detections

\begin{tabular}{|c|c|c|c|c|c|c|c|c|c|c|c|c|}
\hline $\begin{array}{l}\text { Src } \\
\#\end{array}$ & $\begin{array}{l}\text { Peak }^{\mathrm{a}} \\
\quad(\mathrm{mJy} \mathrm{b}\end{array}$ & $\begin{array}{c}P_{\mathrm{err}}^{\mathrm{a}} \\
\left.\mathrm{n}^{-1}\right)\end{array}$ & Total $^{\mathrm{a}}$ & $T_{\mathrm{err}}^{\mathrm{a}}$ & $\begin{array}{c}\mathrm{FWHM}_{a}{ }^{\mathrm{a}} \\
(\operatorname{arcsec})\end{array}$ & $\begin{array}{l}\mathrm{FWHM}_{b}{ }^{\mathrm{a}} \\
(\operatorname{arcsec})\end{array}$ & $\begin{array}{l}\text { P.A. } \\
\text { (deg) }\end{array}$ & $\lim ^{b}$ & $\begin{array}{c}\mathrm{FWHM}_{a, d}{ }^{\mathrm{b}} \\
\quad(\operatorname{arcsec})\end{array}$ & $\begin{array}{c}\mathrm{FWHM}_{b, d}{ }^{\mathrm{b}} \\
\quad(\operatorname{arcsec})\end{array}$ & $\begin{array}{l}\text { P.A. }{ }^{\mathrm{b}} \\
(\mathrm{deg})\end{array}$ & $\begin{array}{l}\text { P.A. } d, e^{\mathrm{b}} \\
\quad(\mathrm{deg})\end{array}$ \\
\hline 1 & 27.25 & 0.35 & 27.69 & 0.65 & 3.613 & 1.855 & 71.2 & $<$ & 0.70 & 0.40 & -1 & -1 \\
\hline 2 & 14.46 & 0.29 & 15.19 & 0.57 & 3.712 & 1.887 & 71.1 & $\ldots$ & 0.74 & 0.42 & 53 & 21 \\
\hline 3 & 5.96 & 0.28 & 5.44 & 0.50 & 3.634 & 1.673 & 75.8 & $<$ & -1.00 & -1.00 & -1 & -1 \\
\hline 4 & 3.48 & 0.67 & 4.30 & 1.40 & 4.240 & 2.400 & 77.0 & $\ldots$ & 1.80 & 0.80 & 107 & 44 \\
\hline 5 & 30.71 & 0.63 & 31.00 & 1.20 & 3.928 & 2.104 & 71.3 & $<$ & 1.00 & 0.40 & -1 & -1 \\
\hline 6 & 49.25 & 0.73 & 52.00 & 1.40 & 3.990 & 2.131 & 71.6 & $\ldots$ & 0.66 & 0.51 & 14 & 65 \\
\hline 7 & 9.17 & 0.44 & 8.95 & 0.80 & 3.452 & 1.855 & 66.1 & $<$ & -1.00 & -1.00 & -1 & -1 \\
\hline 8 & 11.24 & 0.44 & 11.24 & 0.82 & 3.547 & 1.850 & 69.2 & $<$ & -1.00 & -1.00 & -1 & -1 \\
\hline 9 & 59.82 & 0.47 & 78.70 & 1.00 & 3.948 & 2.186 & 73.1 & $\ldots$ & 1.68 & 1.15 & 87 & 6 \\
\hline 10 & 7.10 & 0.44 & 7.56 & 0.86 & 3.744 & 1.866 & 71.3 & $\ldots$ & 1.08 & 0.36 & 76 & 12 \\
\hline 11 & 23.13 & 0.46 & 28.75 & 0.98 & 3.913 & 2.084 & 73.5 & $\ldots$ & 1.61 & 0.93 & 89 & 17 \\
\hline 12 & 11.22 & 0.44 & 11.88 & 0.86 & 3.736 & 1.860 & 69.6 & $\ldots$ & 1.07 & 0.25 & 58 & 14 \\
\hline 13 & 10.95 & 0.15 & 12.04 & 0.30 & 3.939 & 2.182 & 73.2 & $\ldots$ & 0.92 & 0.47 & 137 & 45 \\
\hline 14 & 16.83 & 0.66 & 16.90 & 1.20 & 3.428 & 1.749 & 70.6 & $<$ & 1.10 & 0.40 & -1 & -1 \\
\hline 15 & 36.27 & 0.67 & 43.40 & 1.40 & 4.001 & 2.379 & 76.4 & $\ldots$ & 1.42 & 0.61 & 129 & 19 \\
\hline 16 & 29.64 & 0.67 & 31.20 & 1.30 & 3.830 & 2.138 & 70.4 & $\ldots$ & 0.69 & 0.34 & 9 & 66 \\
\hline 17 & 7.22 & 0.39 & 7.97 & 0.75 & 3.492 & 1.907 & 62.3 & $<$ & -1.00 & -1.00 & -1 & -1 \\
\hline 18 & 9.54 & 0.64 & 9.80 & 1.20 & 3.740 & 2.110 & 72.4 & $<$ & 1.60 & 0.80 & -1 & -1 \\
\hline 19 & 8.20 & 0.63 & 9.20 & 1.30 & 4.230 & 2.040 & 76.6 & $<$ & 2.90 & 0.70 & -1 & -1 \\
\hline 20 & 9.87 & 0.13 & 9.92 & 0.25 & 3.612 & 1.804 & 72.8 & $<$ & 0.90 & 0.20 & -1 & -1 \\
\hline 21 & 4.47 & 0.14 & 5.00 & 0.28 & 3.649 & 1.986 & 70.7 & $\ldots$ & 0.94 & 0.52 & 19 & 51 \\
\hline 22 & 0.61 & 0.12 & 5.10 & 1.10 & 11.050 & 4.900 & 63.4 & $\ldots$ & 10.50 & 4.50 & 62 & 11 \\
\hline 23 & 8.38 & 0.65 & 8.50 & 1.20 & 3.427 & 1.749 & 71.6 & $\ldots$ & 0.56 & 0.17 & 81 & 21 \\
\hline 24 & 3.07 & 0.57 & 2.64 & 0.99 & 3.280 & 1.550 & 73.0 & $<$ & -1.00 & -1.00 & -1 & -1 \\
\hline 25 & 5.56 & 0.64 & 6.80 & 1.30 & 3.640 & 1.980 & 76.7 & $\ldots$ & 1.52 & 0.62 & 105 & 33 \\
\hline 26 & 4.34 & 0.68 & 4.90 & 1.30 & 3.480 & 1.900 & 72.0 & $\ldots$ & 0.86 & 0.73 & 108 & 55 \\
\hline 27 & 2.31 & 0.65 & 2.70 & 1.40 & 3.940 & 1.770 & 76.6 & $<$ & 4.50 & 1.20 & -1 & -1 \\
\hline 28 & 2.49 & 0.13 & 2.67 & 0.25 & 3.602 & 1.908 & 68.1 & $<$ & 1.70 & 0.80 & -1 & -1 \\
\hline 29 & 1.39 & 0.36 & 1.43 & 0.66 & 3.290 & 1.910 & 33.0 & $<$ & -1.00 & -1.00 & -1 & -1 \\
\hline 30 & 15.88 & 0.36 & 17.06 & 0.68 & 3.444 & 1.903 & 70.6 & $\ldots$ & 0.74 & 0.35 & 15 & 53 \\
\hline 31 & 2.35 & 0.15 & 3.05 & 0.32 & 3.835 & 1.955 & 72.3 & $\ldots$ & 1.89 & 0.89 & 75 & 46 \\
\hline 32 & 50.77 & 0.61 & 51.80 & 1.10 & 3.770 & 2.108 & 72.8 & $<$ & 0.70 & 0.50 & -1 & -1 \\
\hline 33 & 9.22 & 0.69 & 10.60 & 1.40 & 3.842 & 1.752 & 67.8 & $<$ & 2.70 & 0.60 & -1 & -1 \\
\hline 34 & 12.23 & 0.20 & 14.24 & 0.40 & 4.041 & 2.294 & 67.6 & $\ldots$ & 1.70 & 0.60 & 50 & 9 \\
\hline 35 & 294.60 & 3.70 & 328.10 & 7.20 & 4.065 & 2.205 & 73.1 & $\ldots$ & 1.33 & 0.67 & 81 & 14 \\
\hline 36 & 129.60 & 4.10 & 246.00 & 11.00 & 4.506 & 3.386 & 58.1 & $\ldots$ & 2.96 & 1.97 & 11 & 15 \\
\hline 37 & 0.70 & 0.14 & 7.30 & 1.60 & 10.300 & 6.000 & 4.8 & $\ldots$ & 10.10 & 5.10 & 2 & 14 \\
\hline 38 & 11.13 & 0.14 & 10.90 & 0.26 & 3.836 & 2.089 & 72.1 & $<$ & 0.30 & 0.10 & -1 & -1 \\
\hline
\end{tabular}

Notes.

${ }^{a}$ Properties of the best-fit Gaussian to the ALMA emission: peak flux, total flux, FWHM of the major and minor axes, and position angle.

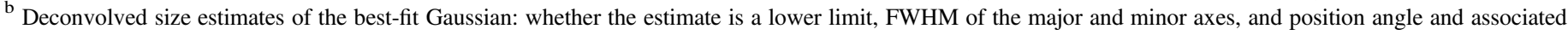
error. Unresolved sources have FWHM and position angle values of -1 .

(near source 11) is clearly visible. There appear to be three roughly equally spaced fragments along this ridge, as was also observed by Nakamura et al. (2012) using data from the Submillimeter Array. This complex emission structure is reminiscent of that predicted by the turbulent fragmentation scenario.

Friesen et al. (2014) observed $\mathrm{H}_{2} \mathrm{D}^{+}$and continuum emission around 360 and $370 \mathrm{GHz}$ with ALMA (Band 7) around SM1N and SM1 (the northern and middle of the three fragments visible in our Figure 5), finding emission in both locations. Their continuum peak in SM1 matches our detection of source 11. Friesen et al. (2014) do not report extended continuum emission in either SM1N or SM1, but their observations have a smaller maximum angular scale $\left(\sim 6^{\prime \prime}\right)$, which suggests that the emission which we see would have been filtered out. Friesen et al. (2014) suggest that the compact emission around SM1 is most likely attributable to a warm accretion disk around a very young protostar. Meanwhile, SM1N is more likely to be still starless, although the presence of a low-luminosity protostar is also possible. The extended emission that we detect around SM1N is therefore a candidate for an additional starless core detection.

The second mosaic where faint extended emission is visible is shown in Figure 6. Both the extended emission and a compact source (source 28) are in close proximity to a Class Flat YSO. Examination of the Herschel data in this field suggests that the extended emission is associated with warm dust that is being heated by the neighboring protostar. 
Table 4

Physical Properties of Detections

\begin{tabular}{|c|c|c|c|c|c|}
\hline $\begin{array}{l}\mathrm{Src}^{\mathrm{a}} \\
\text { Num }\end{array}$ & $\begin{array}{c}\text { Mass }^{\mathrm{b}} \\
\left(M_{\odot}\right)\end{array}$ & & $\begin{array}{l}R_{\text {eff }}^{\mathrm{b}} \\
\text { (au) }\end{array}$ & & $\begin{array}{c}n^{\mathrm{b}} \\
\left(\mathrm{cm}^{-3}\right)\end{array}$ \\
\hline 1 & 0.2602 & $<$ & 37 & $>$ & $1.83 \mathrm{E}+11$ \\
\hline 2 & 0.1427 & $\ldots$ & 39 & $\ldots$ & $8.61 \mathrm{E}+10$ \\
\hline 3 & 0.0511 & $<$ & 788 & $>$ & $3.74 \mathrm{E}+06$ \\
\hline 4 & 0.0404 & $\ldots$ & 83 & $\ldots$ & $2.44 \mathrm{E}+09$ \\
\hline 5 & 0.2913 & $<$ & 44 & $>$ & $1.20 \mathrm{E}+11$ \\
\hline 6 & 0.4885 & $\ldots$ & 40 & $\ldots$ & $2.61 \mathrm{E}+11$ \\
\hline 7 & 0.0841 & $<$ & 775 & $>$ & $6.47 \mathrm{E}+06$ \\
\hline 8 & 0.1056 & $<$ & 792 & $>$ & $7.62 \mathrm{E}+06$ \\
\hline 9 & 0.7394 & $\ldots$ & 97 & $\ldots$ & $2.88 \mathrm{E}+10$ \\
\hline 10 & 0.0710 & $\ldots$ & 43 & $\ldots$ & $3.06 \mathrm{E}+10$ \\
\hline 11 & 0.2701 & $\ldots$ & 85 & $\ldots$ & $1.54 \mathrm{E}+10$ \\
\hline 12 & 0.1116 & $\ldots$ & 36 & $\ldots$ & $8.43 \mathrm{E}+10$ \\
\hline 13 & 0.1131 & $\ldots$ & 46 & $\ldots$ & $4.16 \mathrm{E}+10$ \\
\hline 14 & 0.1588 & $<$ & 46 & $>$ & $5.68 \mathrm{E}+10$ \\
\hline 15 & 0.4078 & $\ldots$ & 65 & $\ldots$ & $5.29 \mathrm{E}+10$ \\
\hline 16 & 0.2931 & $\ldots$ & 33 & $\ldots$ & $2.70 \mathrm{E}+11$ \\
\hline 17 & 0.0749 & $<$ & 762 & $>$ & $6.04 \mathrm{E}+06$ \\
\hline 18 & 0.0921 & $<$ & 79 & $>$ & $6.64 \mathrm{E}+09$ \\
\hline 19 & 0.0864 & $<$ & 99 & $>$ & $3.12 \mathrm{E}+09$ \\
\hline 20 & 0.0932 & $<$ & 29 & $>$ & $1.28 \mathrm{E}+11$ \\
\hline 21 & 0.0470 & $\ldots$ & 48 & $\ldots$ & $1.44 \mathrm{E}+10$ \\
\hline 22 & 0.0479 & $\ldots$ & 481 & $\ldots$ & $1.54 \mathrm{E}+07$ \\
\hline 23 & 0.0799 & $\ldots$ & 21 & $\ldots$ & $2.84 \mathrm{E}+11$ \\
\hline 24 & 0.0248 & $<$ & 744 & $>$ & $2.15 E+06$ \\
\hline 25 & 0.0639 & $\ldots$ & 67 & $\ldots$ & $7.30 \mathrm{E}+09$ \\
\hline 26 & 0.0460 & $\ldots$ & 55 & $\ldots$ & $9.67 \mathrm{E}+09$ \\
\hline 27 & 0.0254 & $<$ & 162 & $>$ & $2.11 \mathrm{E}+08$ \\
\hline 28 & 0.0251 & $<$ & 81 & $>$ & $1.65 \mathrm{E}+09$ \\
\hline 29 & 0.0134 & $<$ & 555 & $>$ & $2.81 \mathrm{E}+06$ \\
\hline 30 & 0.1603 & $\ldots$ & 35 & $\ldots$ & $1.27 \mathrm{E}+11$ \\
\hline 31 & 0.0287 & $\ldots$ & 90 & $\ldots$ & $1.37 \mathrm{E}+09$ \\
\hline 32 & 0.4867 & $<$ & 41 & $>$ & $2.46 \mathrm{E}+11$ \\
\hline 33 & 0.0996 & $<$ & 89 & $>$ & $5.05 \mathrm{E}+09$ \\
\hline 34 & 0.1338 & $\ldots$ & 70 & $\ldots$ & $1.36 \mathrm{E}+10$ \\
\hline 35 & 3.0826 & $\ldots$ & 66 & $\ldots$ & $3.83 \mathrm{E}+11$ \\
\hline 36 & 2.3112 & $\ldots$ & 169 & $\ldots$ & $1.72 \mathrm{E}+10$ \\
\hline 37 & 0.0686 & $\ldots$ & 502 & $\ldots$ & $1.94 \mathrm{E}+07$ \\
\hline 38 & 0.1024 & $<$ & 12 & $>$ & $2.06 \mathrm{E}+12$ \\
\hline
\end{tabular}

Notes.

a Source number, from Table 2.

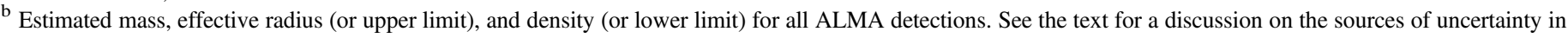
the mass estimates.

\section{Properties of the Detections}

\subsection{SCUBA Core Concentration}

The central concentrations, or measures of the peakiness, of the SCUBA dense cores we targeted were determined by Jørgensen et al. (2008). Concentration is defined as

$$
C=1-\frac{1.13 B^{2} F_{\mathrm{tot}}}{\pi R^{2} F_{\mathrm{pk}}}
$$

where $B$ is the beamsize, $F_{\text {tot }}$ is the total flux, $R$ is the radius, and $F_{\mathrm{pk}}$ is the peak flux (Johnstone et al. 2000). A histogram of the SCUBA core concentrations is shown in Figure 7. Although there is a wide range of concentrations in both the protostellar and starless core populations, most of the high- concentration cores tend to be protostellar. In their analysis of the Perseus molecular cloud, Jørgensen et al. (2007) found that all cores with concentrations above 0.6 were associated with Spitzer-identified protostars. This same trend, however, did not hold in their similar analysis of Ophiuchus, and Jørgensen et al. (2008) identified there four apparently starless cores with high concentrations.

Source 37, our starless core detection shown in Figure 4, is found in one of these four high-concentration SCUBA starless cores, with a concentration of 0.74 measured in Jørgensen et al. (2008). The remaining three high-concentration SCUBA starless cores all lie within the mosaic shown in Figure 5. The first of these is associated with ALMA source 10 , a detection where $\mathrm{X}$-ray observations suggest there is a 


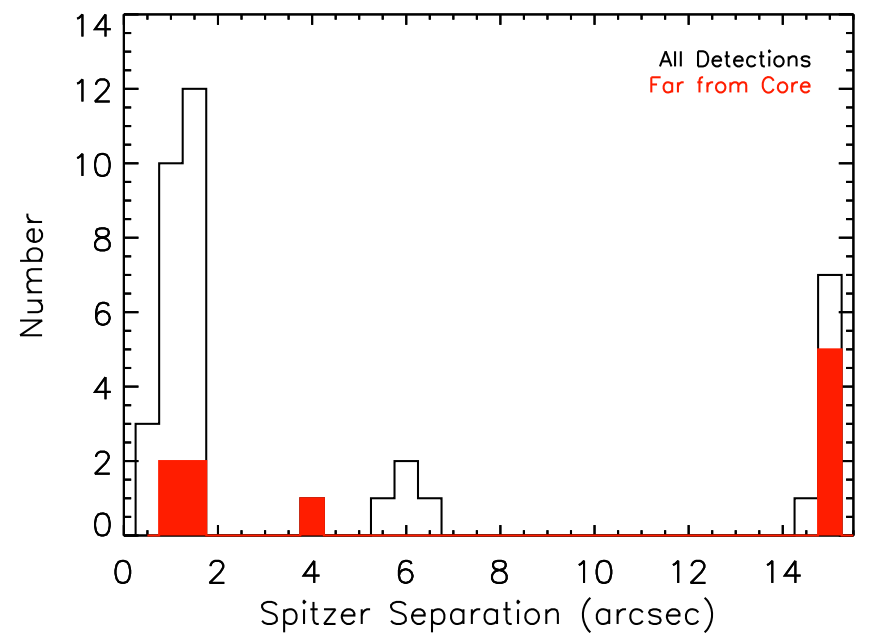

Figure 3. Distribution of angular separations between ALMA detections and the nearest Spitzer YSO. The full sample is shown by the black outline, while ALMA detections which are highly separated from their nearest SCUBA core peak $\left(>15^{\prime \prime}\right)$ are shown in the filled red histogram. All sources with Spitzer separations larger than $15^{\prime \prime}$ are included in the final bin.

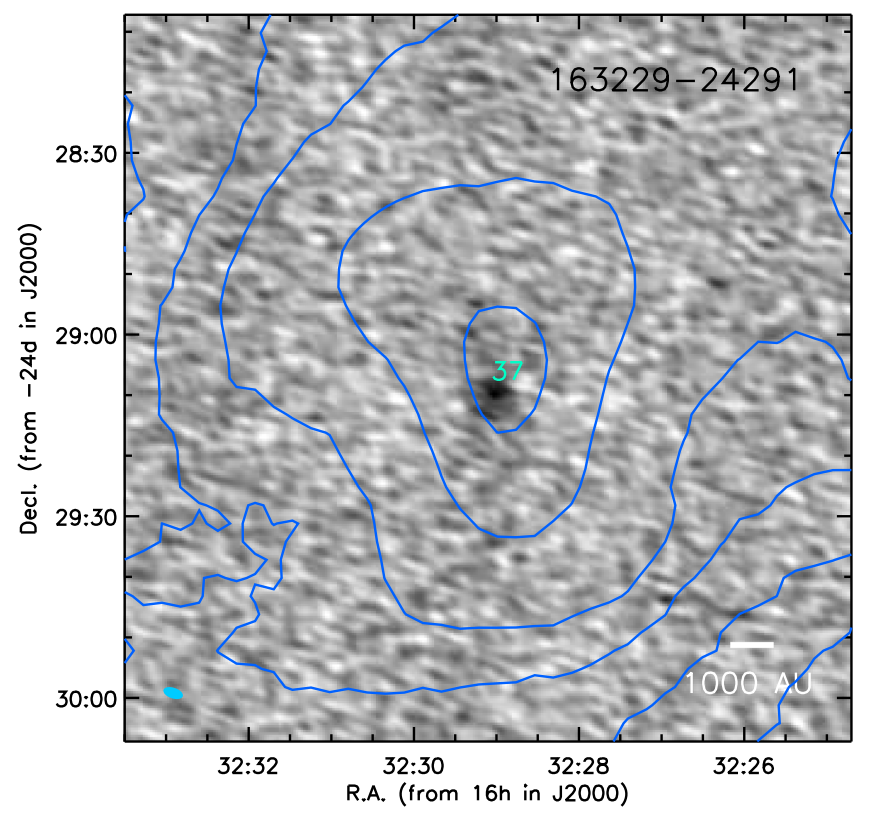

Figure 4. ALMA single pointing detection of source 37, a starless core in field 163229-24291. The grayscale ranges from -0.5 to $1 \mathrm{mJy}^{\text {beam }}{ }^{-1}$, and the beam is shown in the bottom left corner. Blue contours show SCUBA-2 $850 \mu \mathrm{m}$ emission at levels of $0.15 \mathrm{mJy}$ sq. $\operatorname{arcsec}{ }^{-1}, 0.5 \mathrm{mJy}$ sq. $\operatorname{arcsec}^{-1}, 1 \mathrm{mJy} \mathrm{sq} . \operatorname{arcsec}^{-1}$, $1.5 \mathrm{mJy}$ sq. $\operatorname{arcsec}^{-1}, 3 \mathrm{mJy}$ sq. $\operatorname{arcsec}^{-1}$, and $5 \mathrm{mJy}$ sq. $\operatorname{arcsec}^{-1}$. The horizontal line indicates a length scale of 1000 au for a distance to Ophiuchus of $140 \mathrm{pc}$. Compact sources of emission detected are labeled with their number given in Table 2. This detection coincides with the starless core L1689N from Lis et al. (2016), while the closest known protostar is IRAS 16293-2422, which lies outside of the area plotted.

faint hidden protostar. The second is core 162627-24233, the northernmost core in the Figure 5 mosaic. Although ALMA did not detect any emission near the SCUBA core's peak, it did find two sources of compact emission significantly offset to the west and east of the SCUBA peak, sources 7 and 12 respectively. Source 7 is associated with a Dunham et al. (2015) Spitzer YSO, while source 12 is associated with a

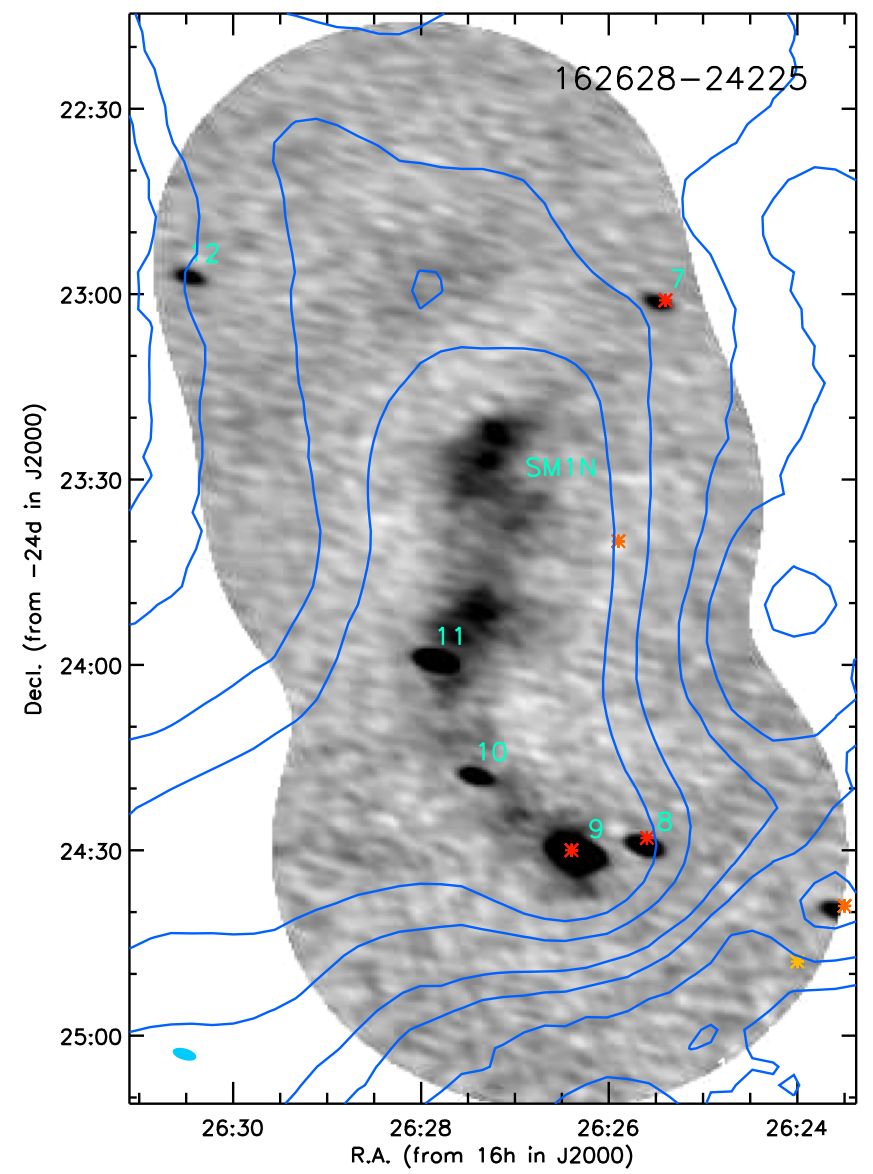

Figure 5. ALMA mosaic with diffuse extended emission which appears somewhat filamentary. All compact ALMA detections in the field are associated with protostellar sources. See Figure 4 for the plotting conventions adopted. In this and subsequent figures, the grayscale ranges from -1 to $2 \mathrm{mJy}$ beam $^{-1}$. Colored asterisks show Spitzer YSOs from Dunham et al. (2015), with shading ranging from red for Class $0 /$ I to yellow for Class II. The compact emission in the approximate center of the mosaic (near source 11) coincides with a compact source seen in ALMA Band 7 continuum emission by Friesen et al. (2014), a source known as SM1. Friesen et al. (2014) also detect compact emission in the vicinity of the diffuse emission directly east of the SM1N label; the compact emission may be obscured in our map by the bright diffuse emission present. The southernmost two Class 0/I ALMA detections (sources 8 and 9) are VLA 1623 and VLA $1623 \mathrm{~W}$.

Spitzer YSOc source in the full uncut Spitzer c2d catalog (see Section 3.3). The final high-concentration SCUBA starless core in Figure 5 is not directly associated with any of the compact emission sources seen with ALMA; however, it does contain the extended emission seen around SM1N discussed earlier.

Even if these high concentrations do not imply the presence of hidden faint protostars, they are expected to be indicative of more-evolved cores, with the presence of a more concentrated peak of emission expected to occur near the time when a starless core begins to collapse to form a protostar. Our observations reinforce this picture. None of the SCUBA starless cores with concentrations below 0.6 were detected with ALMA, while all four of the starless cores with concentrations above 0.6 have at least one associated detection, and two of these four "starless" cores (associated with ALMA sources 10 and 12) may already harbour faint protostars. 


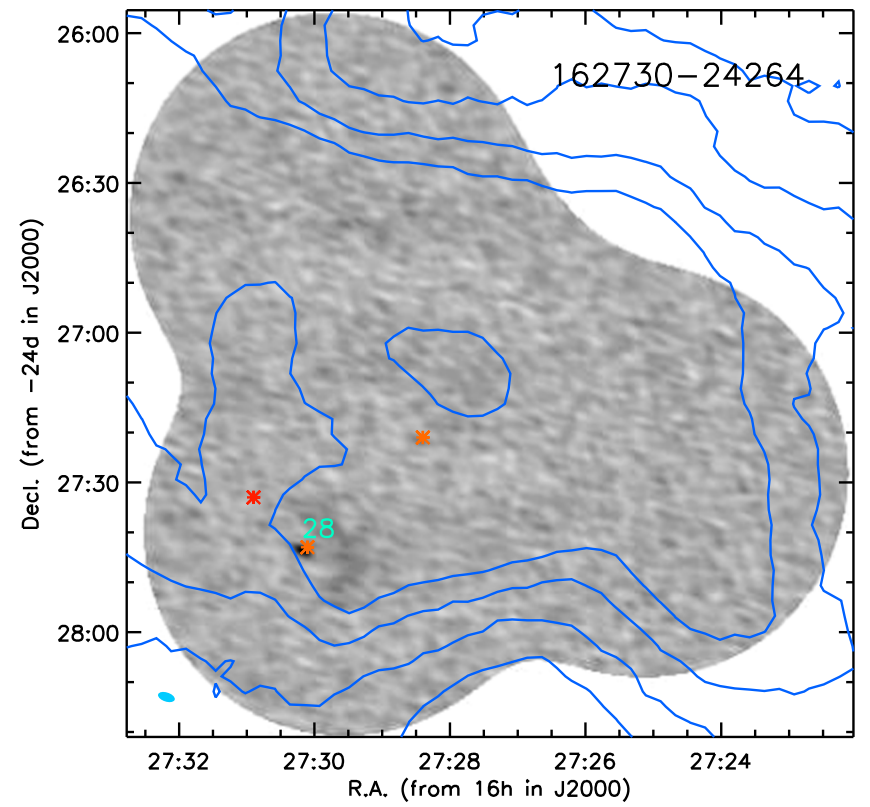

Figure 6. ALMA mosaic showing both a compact emission source (source 28) and faint extended emission in the southeast area of the map. The extended emission appears to be warm dust which is being heated by the neighboring protostar. See Figures 4 and 5 for the plotting conventions used.

\subsection{ALMA Peak Fluxes}

In Figure 8, we show the distribution of peak fluxes measured for the ALMA detections. The filled blue histogram shows source 37 which appears to be starless. Notably, source 37 lies in the lowest peak flux bin. Since starless cores are expected to pass through higher-density phases more rapidly (see the discussion in the next section), it is reassuring to see source 37 lying at the low end of the distribution.

We note that if the diffuse emission we see around SM1N were included as a source in Figure 8, it would lie near source 37 with a peak flux around $2 \mathrm{mJy}^{\text {beam }}{ }^{-1}$.

\section{Substructure from Turbulent Fragmentation}

We now examine the number of starless cores that we would expect to detect under the turbulent fragmentation picture. For this analysis, we take a similar approach to that outlined in D16. The premise is that numerical simulations of the evolution of dense cores may provide a better guide to the detectability of substructure within dense cores than would a simple model such as a collapsing Bonnor-Ebert sphere model. D16 also analyze the detectability of a collapsing Bonnor-Ebert sphere, and find that it spends little time in a highly dense and concentrated state. Over an ensemble of cores at different ages, a smaller fraction of Bonnor-Ebert spheres should be detectable than substructure generated in the numerical simulations including turbulence and/or magnetic fields.

D16 analyze simulations of isolated collapsing prestellar cores performed using the ORION adaptive mesh refinement code (Li et al. 2012). The calculations include turbulence, an initially uniform vertical magnetic field, and self-gravity. Further evolution of cores with these initial conditions finds

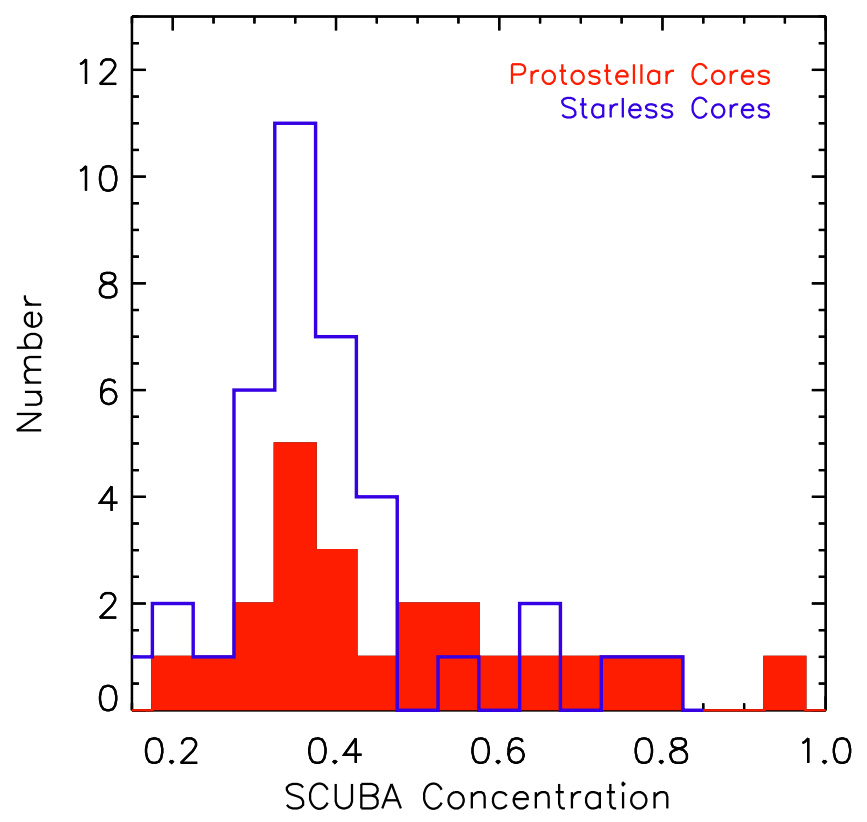

Figure 7. Distribution of concentrations measured for dense cores within Ophiuchus. The dark blue line indicates cores that we classify as starless, while the filled red histogram indicates cores that we classify as protostellar.

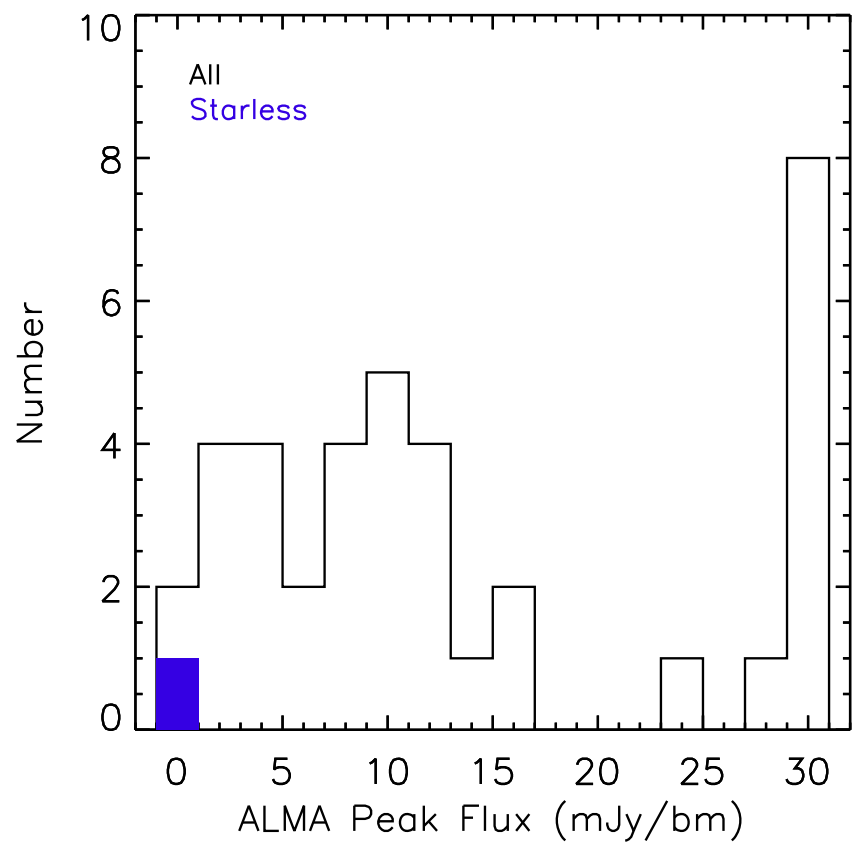

Figure 8. Distribution of peak fluxes of ALMA detections. Sources with peak fluxes higher than the maximum bin size plotted are included in the final bin. The black empty histogram shows all 38 ALMA detections, while the filled blue histogram shows source 37 , the starless core detection.

that secondary fragmentation, leading to wide-binary formation, is common (Offner et al. 2016). D16, however, only perform the analysis until the first star (sink particle) forms, at which point collapse has produced substructure but not multiplicity. Using synthetic observations of these simulations, D16 demonstrate 


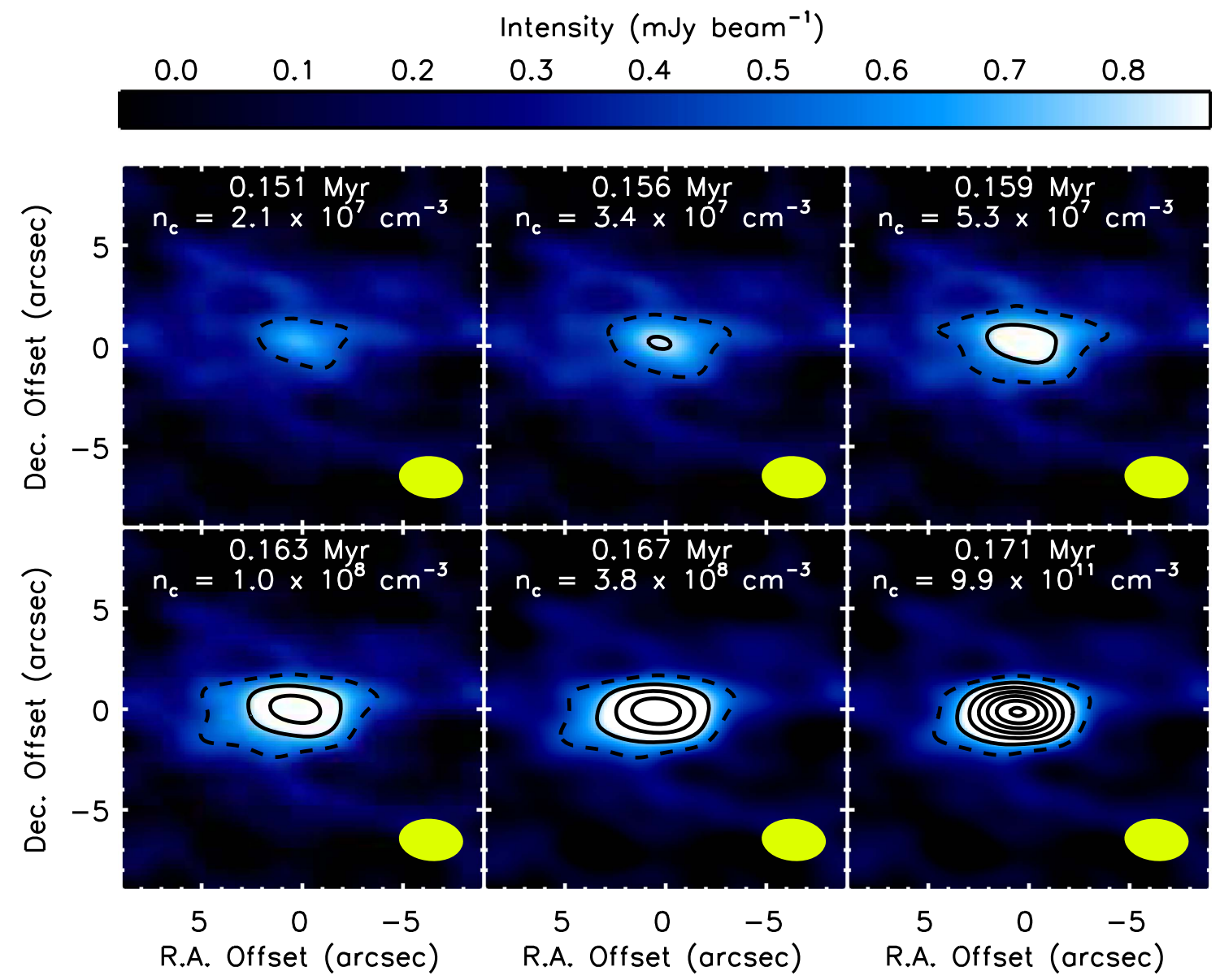

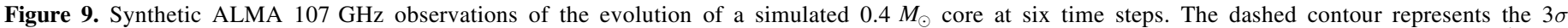

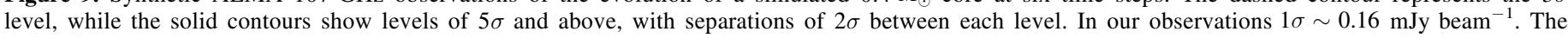

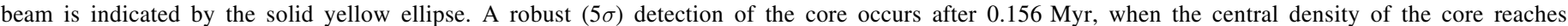
$3.4 \times 10^{7} \mathrm{~cm}^{-3}$.

that for their ALMA Cycle 1 setup they expect to detect cores with central densities above $8.9 \times 10^{7} \mathrm{~cm}^{-3}$. This value is derived based on synthetic observations of a dense core with a total mass of $0.4 M_{\odot}$; a higher-mass core $\left(4 M_{\odot}\right)$ would be detectable when its central density exceeds $3.3 \times 10^{7} \mathrm{~cm}^{-3}$. We note the simulation core masses are upper limits compared to the observations, since they do not account for observational detection limits or consider how SCUBA cores are defined.

The detection threshold is also a function of both the sensitivity of the array and the spatial filtering implied by the antenna configuration. We therefore re-run the synthetic observations presented in D16 to account for the antenna configuration used for our Ophiuchus observations, in addition to scaling the simulations to the slightly closer distance of $140 \mathrm{pc}$ for Ophiuchus (compared with $150 \mathrm{pc}$ for Chamaeleon). D16 used a column densitytemperature relationship derived from Bonnor-Ebert sphere models to estimate the total observable flux implied by the simulations. The core interiors that we observe are heavily shielded from the radiation field impinging on the molecular cloud exterior. The temperatures on the sub-core scales of interest should therefore be similar in both Chamaeleon and Ophiuchus, and we apply the same relationship as in D16.

In Ophiuchus, adjusting the Jørgensen et al. (2008) core catalog values to our assumed distance of $140 \mathrm{pc}$, we find a median core mass of $0.55 M_{\odot}$. Hence, as in D16, the $0.4 M_{\odot}$ core is the most applicable simulation to use for the synthetic observations.

Figure 9 shows our synthetic ALMA observations of the $0.4 M_{\odot}$ core simulation, adopting a distance of $140 \mathrm{pc}$ and the antenna configuration used for our real observations. Similar to the synthetic observations generated by D16, the synthetic observations were generated using the CASA tasks SIMOBSERVE and SIMANALYZE. We assigned a position of R.A. $=16: 30: 00$ and decl. $=-24: 30: 00$, to the simulations, representing the middle of the area observed in Ophiuchus. We assumed $1 \mathrm{~s}$ integration times and included thermal noise from the atmosphere, for a total integration time of $60 \mathrm{~s}$, with an effective mean frequency of $107 \mathrm{GHz}$ and bandwidth of $6 \mathrm{GHz}$. We cleaned to a threshold of $0.30 \mathrm{mJy} \mathrm{beam}^{-1}$, i.e., approximately $2 \sigma$, using non-interactive cleaning with no clean mask set a priori. The synthetic observations do not account for the flagging applied to the real observations, which was extensive in our data, nor the exact elevation or weather conditions that we observed under. Thus, the synthetic observations have a somewhat lower noise level than our real observations do. We only applied clean and searched for detections in terms of our typical observed noise level to account for this difference in data quality. 
As can be seen in Figure 9, the central core peak is only detected in the simulations at a $5 \sigma$ level after $0.156 \mathrm{Myr}$, when the central density of the core reaches $3.7 \times 10^{7} \mathrm{~cm}^{-3}$. This is an earlier and less dense stage of the core's evolution than D16 found for the first core detection in Chamaeleon-like cores. The primary driver for this difference arises from our observations having more antennae at shorter baselines, improving the sensitivity to larger-scale structure.

In order to predict the number of cores detected based on the observational set-up, D16 then assumed that the lifetime of a dense core varies with its freefall time (see, e.g., Jessop \& Ward-Thompson 2000). Under the important assumption that star formation is continuous in the region, with the dense core lifetime at different densities described by the freefall time, D16 express the number of ALMA detections expected as

$$
\text { Detections }>\frac{2}{3} \times N_{\text {total }} \times\left(\frac{n_{\text {Detectable }}}{n_{\text {Limit }}}\right)^{-0.5}
$$

where $N_{\text {total }}$ is the number of starless cores observed, $n_{\text {Detectable }}$ is the density at which ALMA is able to detect the starless cores, and $n_{\text {Limit }}$ is the observed lower limit of the mean core densities as observed at single-dish resolution. The factor of two thirds arises from the fact that D16 note that in the simulations, detections are much more difficult for cores viewed along the local magnetic field axis. In the distanceadjusted Jørgensen et al. (2008) catalog, the minimum core density to apply in Equation (4) is $2.5 \times 10^{5} \mathrm{~cm}^{-3}$, for our adopted temperature and distance, while the mean core density is $1.5 \times 10^{6} \mathrm{~cm}^{-3}$.

For Ophiuchus, we have $N_{\text {total }}=37$ starless cores ${ }^{13}$ with a minimum density of $n_{\text {Limit }}=2.5 \times 10^{5} \mathrm{~cm}^{-3}$, which we expect are detectable with our ALMA setup down to a density of $n_{\text {Detectable }}=3.7 \times 10^{7} \mathrm{~cm}^{-3}$. Based on Equation (4), we would therefore predict a minimum of 2.1 (two) starless cores to have detectable substructure. D16 additionally demonstrated the much greater difficulty of detecting Bonnor-Ebert spheres compared with the simulated starless cores, when both were observed under identical conditions. D16 found that a Bonnor-Ebert sphere was not detectable until it had a central density more than 100 times larger than the minimum density threshold for detecting the simulated cores, implying a detection rate more than ten times lower for the Bonnor-Ebert sphere model.

In our observed sample, we have one detection which has strong evidence suggesting that it is not associated with a protostellar core. We also have one detection of extended emission around SM1N which is tentatively unassociated with any YSOs. These detections suggest that the cores are not welldescribed by the simple Bonnor-Ebert sphere model, as under that scenario no detections are expected. Including both sources as starless core detections, we satisfy Equation (4). Considering simple Poisson statistics, the chance of having one or two detections is equally likely ( $27 \%$ in both cases), so even if we

\footnotetext{
${ }^{13}$ The astute reader will note that in Section 3.3, we found seven ALMA detections which had large separations from all YSOs in Dunham et al. (2015) but were classified as protostellar rather than starless based on comparison with ancillary data. For various reasons, including the large offset of many of these detections from the nominal dense core center, and detections which share a common dense core field, re-classifying seven of the ALMA detections as protostellar does not imply that there are an equivalent seven SCUBA starless core targets which should be re-classified as protostellar. Nonetheless, even if we did assume only 30 starless cores, the resulting detection rate from Equation (4) would still be two.
}

only consider one detection, we are not inconsistent with Equation (4).

\subsection{Comparison to Chamaeleon I}

In their search for substructure within starless cores in Chamaeleon I, D16 had no detections, despite expecting at least two based on the arguments discussed in the previous section. The D16 null result had reasonably high statistical significance, and they discussed three possible reasons for the disagreement. The first, which they argued was the most likely, is that star formation is not continuous in Chamaeleon I, i.e., the present population of starless cores is not likely to collapse and form protostars in the near future, and instead may be even on their way to dispersing. Simple virial estimates of cores in Chamaeleon I suggest that most of the starless cores are stable against gravitational collapse, which lends credence to this argument. The remaining two possible reasons for disagreement have more global implications, and would be also expected to manifest themselves in observations of starless cores in other molecular clouds. The second is that the assumption that the core lifetime is proportional to the freefall time is not correct, while the third is that the numerical simulations used for the comparisons are not applicable.

If either of the latter two more global explanations put forward by D16 are true, we could reasonably expect a similar outcome in Ophiuchus, i.e., fewer detections of substructure than the lower limit that Equation (4) predicts. Unless both our one strong and one tentative non-protostellar detections are actually attributable to mis-classified protostellar sources, we have identified as many starless core substructures as expected. This result lends credence to the hypothesis proposed in D16 that the dense cores in Chamaeleon I are in an unusual state of evolution which has contributed to the lack of detections in that cloud.

Properties of the ambient molecular cloud environment may also play a role, and perhaps are tied to the difference between Chamaeleon I and Ophiuchus, and could imply that the simulations also poorly reflect core evolution in a Chamaeleon I-type environment. Comparing core detection statistics in different regions implicitly assumes that cores have similar structures across clouds, which may not be entirely correct. In single-dish observations, for example, dense cores in Ophiuchus tend to appear much more compact and clustered than in other nearby clouds. Dense cores in Ophiuchus show sizes of $14^{\prime \prime}-40^{\prime \prime}$ with the majority having sizes under 30" (Pattle et al. 2015), while dense cores in Chamaeleon I have sizes ranging from $20^{\prime \prime}$ to $140^{\prime \prime}$ with the majority having sizes well above 30" (Belloche et al. 2011). (Recall that the two clouds have similar distances, of $140 \mathrm{pc}$ and $150 \mathrm{pc}$ respectively.) Cores which are typically smaller on single-dish scales could also have a more compact configuration of mass on smaller scales, which would then increase their central densities and likelihood of detection with ALMA. As seen with our Ophiuchus observations, it is also possible that typically smaller and more clustered cores may live in a generally more complex environment where dense substructures can form outside of the most obvious density peaks seen in single-dish observations. 

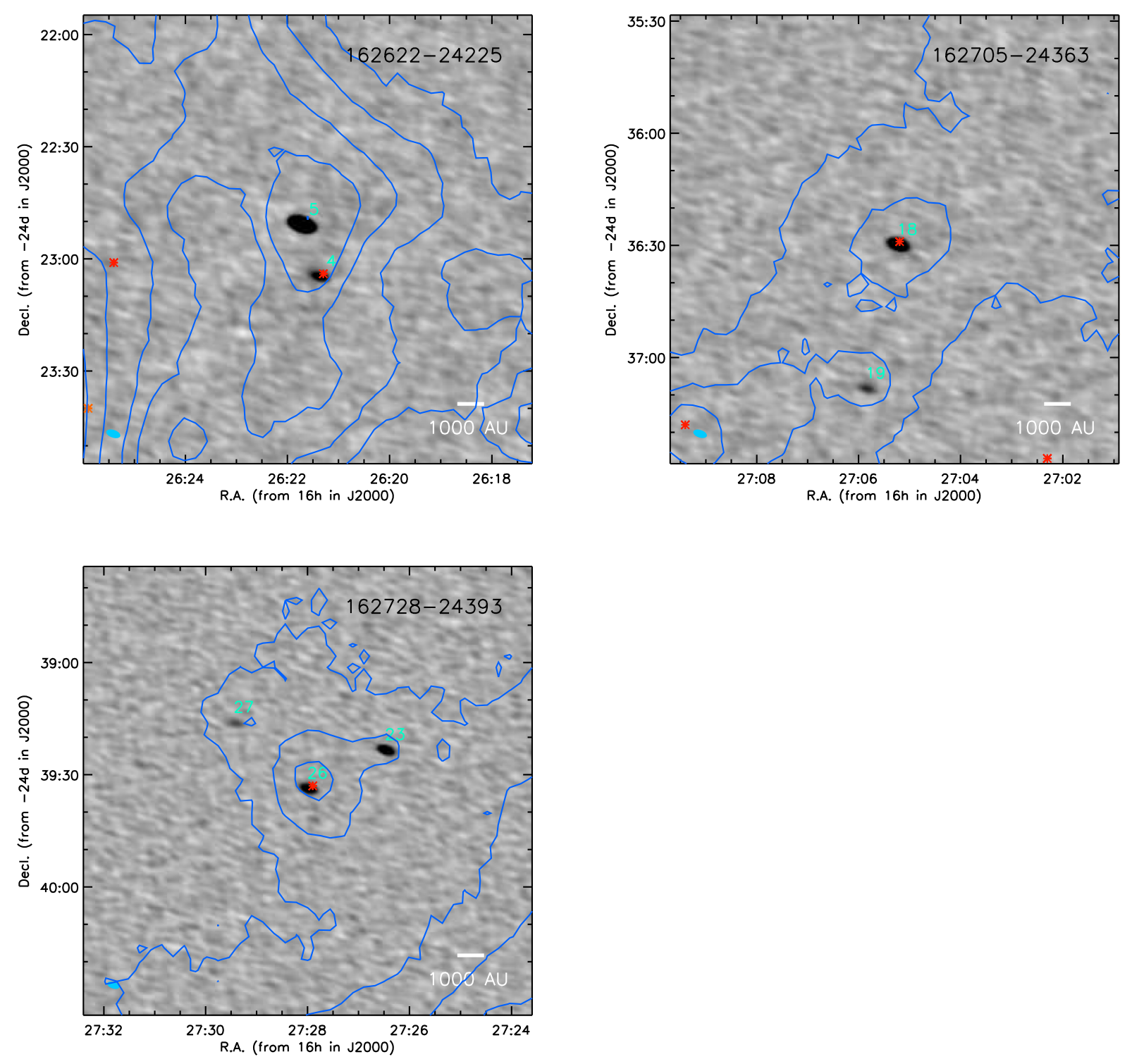

Figure 10. ALMA detections of sources separated by at least $14^{\prime \prime}$ from the nearest Spitzer YSO, but showing other signs of a protostellar nature. See Figures 4 and 5 for the plotting conventions adopted. These sources are source 5, 19,23, and 27. Other close protostellar associations in the field are also labelled.

\subsection{Comparison to Orion}

Kainulainen et al. (2016) also recently examined ALMA continuum observations of the northern portion of the integral shaped filament in Orion. They detected 43 compact sources across a mosaic of the filament, including 18 that do not appear to be associated with a protostar. Given the high degree of nebulosity within Orion, however, it is difficult to be completely certain of a starless classification. The number of expected detections also cannot be easily quantified for this study, as the number and properties of starless cores within the mosaic observed were not quantified prior to the ALMA observations. With an appropriate starless core catalog and synthetic observations of simulations tuned to the Orion data, it would be interesting to see how the Kainulainen et al. (2016) results compare to Ophiuchus and Chamaeleon I.

These ALMA results highlight the fact that starless core populations need to be observed across multiple molecular clouds, and preferably in a uniform manner. This would increase the detection statistics, allowing for more stringent tests of models, and also help to disentangle region-specific behavior that may be occurring.

\section{Conclusion}

We present ALMA Cycle 2 Band 3 (3 mm) observations of 60 dense cores in the Ophiuchus molecular cloud, which were previously identified with SCUBA at the JCMT. We detect 38 compact emission structures with ALMA, including at least one detection which has strong signs of being starless. An additional source of diffuse emission, Oph-SM1N may also be starless. Most of the known protostars falling within our surveyed area were detected, as discussed in more detail in Appendix A.3.

Our ALMA observations should be easily able to detect very low-luminosity protostars or first hydrostatic cores that were undetectable with Spitzer. Indeed, we initially identified eight 

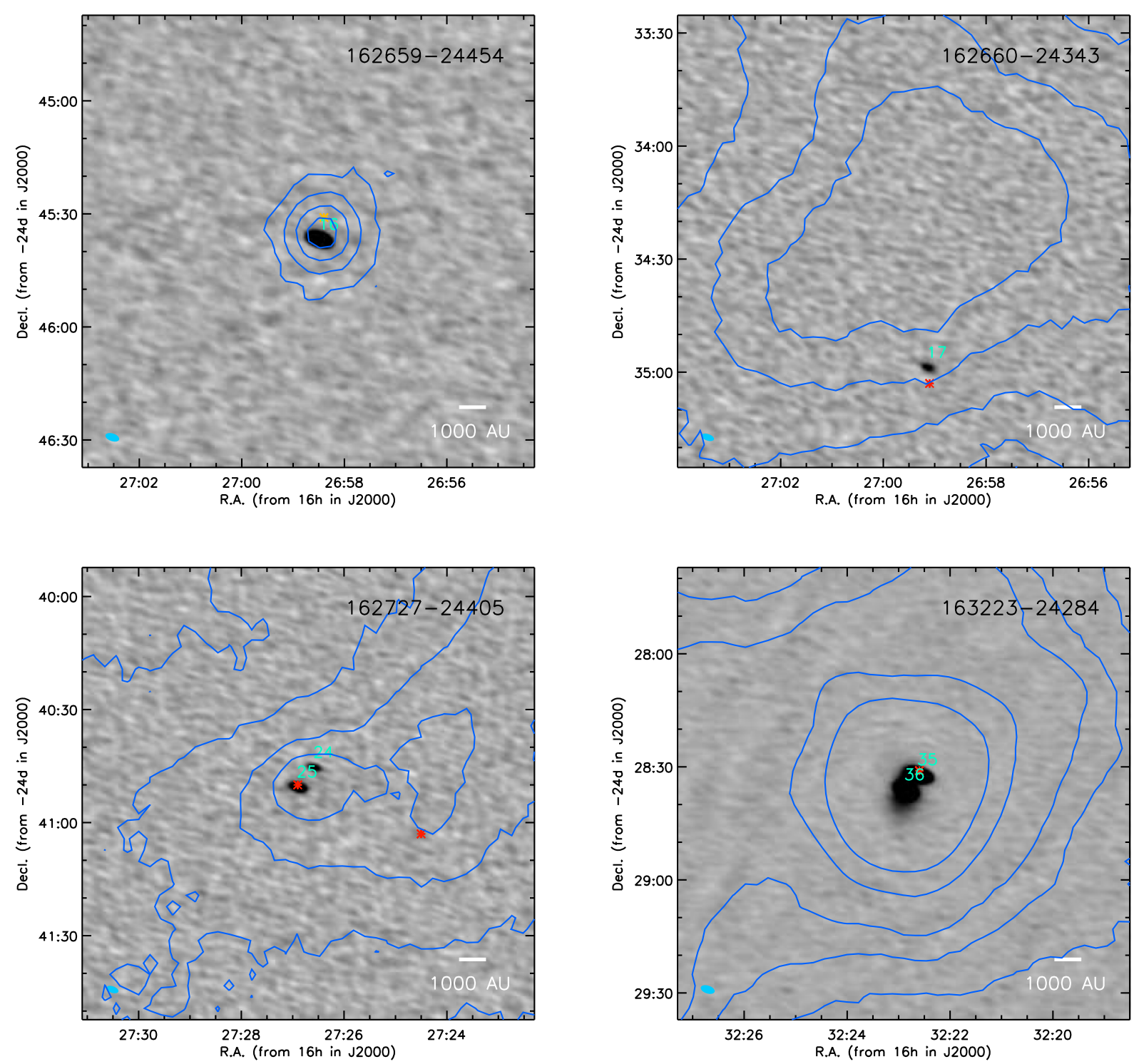

Figure 11. ALMA detections where there is a moderate separation between the detection and the closest Spitzer YSO, but where there are other signs of a protostellar nature. Sources 16, 17, 24, and 36 have moderate Spitzer separations. See Figures 4 and 5 for the plotting conventions adopted. For the field 163223-24284 only, the range for the grayscale image is -10 to $20 \mathrm{mJy}_{\text {beam }}{ }^{-1}$. In the top left panel, Herschel shows a a single protostar lying coincident with ALMA source 16, suggesting that there is a positional error for the Spitzer YSO. Sources 35 and 36 correspond to the binary protostar IRAS 16293-2422; both members of the binary are identified as protostellar in the SIMBAD database.

ALMA detections which had no counterparts in the Dunham et al. (2015) Spitzer YSO catalog. Seven of these detections showed strong evidence of a protostellar nature using ancillary data, leaving just a single strong candidate starless detection. We additionally identify extended emission around Oph SM1N, which we classify as a candidate starless core detection.

One or both of our detections of starless candidates may reveal the substructure present within starless cores near the end of their evolution toward protostars. Following comparisons with synthetic observations of numerical simulations and simple analytic arguments similar to those presented in Dunham et al. (2016), the number of starless core detections predicted $(>2)$ is somewhat consistent with the number we actually detect (one strong candidate and one additional candidate). Both of our candidate starless detections are associated with high-concentration SCUBA cores and are located near a SCUBA core peak, with low ALMA peak flux, as expected for true starless core detections. If the starless cores in Ophiuchus had instead been well-described by a smooth Bonnor-Ebert sphere model, we would have expected no detections. In Chamaeleon I, Dunham et al. (2016) detected no starless cores, despite an expectation of detecting at least two based on a comparison with numerical simulations. We argue that the most likely explanation for the difference between Ophiuchus and Chamaeleon I cores is one put forward by Dunham et al. (2016), namely, that the starless cores presently in Chamaeleon I are not evolving toward a protostellar phase, and currently do not appear to be gravitationally unstable. Observations of additional starless core populations are necessary to confirm whether or not this picture of starless core evolution is correct. As ALMA gains further antennas, new observations should additionally become more sensitive to multiple turbulence-generated fragments (e.g., Offner et al. 2012) if they exist, informing models of stellar multiplicity. 


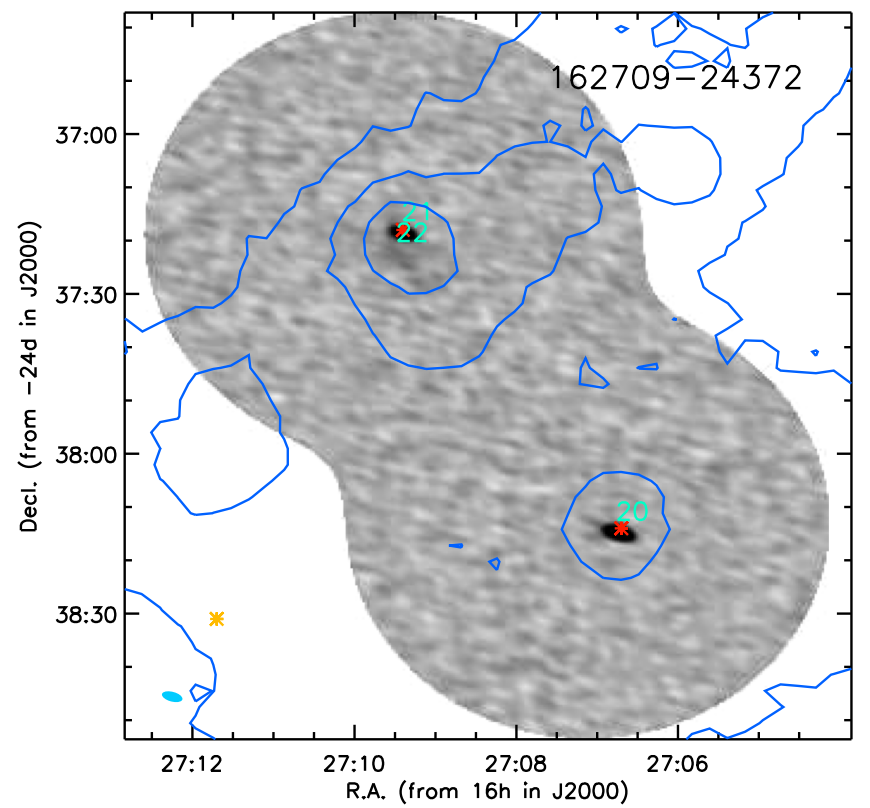

Figure 12. Mosaic containing an ALMA detection where there is a moderate separation between the detection and the closest Spitzer YSO, but there are other signs of a protostellar nature. See Figures 4 and 5 for the plotting conventions adopted. Source 22 is a marginal $(<5 \sigma)$ detection, which has a moderate Spitzer separation. It lies directly south of the bright source 21 .

The authors thank the referee for their careful and constructive comments which improved our paper. The National Radio Astronomy Observatory is a facility of the National Science Foundation operated under cooperative agreement by Associated Universities, Inc. This paper makes use of the following ALMA data: ADS/JAO.ALMA\# 2013.1.00187.S. ALMA is a partnership of ESO (representing its member states), NSF (USA), and NINS (Japan), together with NRC (Canada) and NSC and ASIAA (Taiwan), in cooperation with the Republic of Chile. The Joint ALMA Observatory is operated by ESO, AUI/NRAO, and NAOJ. This research also made use of NASA's Astrophysics Data System (ADS) Abstract Service and the IDL Astronomy Library hosted by the NASA Goddard Space Flight Center. This research has made use of the SIMBAD database, operated at CDS, Strasbourg, France (Wenger et al. 2000). The JCMT has historically been operated by the Joint Astronomy Center on behalf of the Science and Technology Facilities Council of the United Kingdom, the National Research Council of Canada and the Netherlands Organisation for Scientific Research. Additional funds for the construction of SCUBA-2 were provided by the Canada Foundation for Innovation. The identification number for the programme under which the SCUBA-2 data used in this paper is MJLSG32.

Facilities: ALMA, Spitzer, JCMT, Herschel.

Software: CASA.

\section{Appendix A Protostellar Detections}

Here, we show our ALMA protostellar detections, moving through the different Dunham et al. (2015) Spitzer YSO separation regimes in Figure 3 in turn. We emphasize that although some of these ALMA detections have large separations from the nearest Spitzer YSO in the Dunham et al. (2015) catalog, all show signs of a protostellar nature in ancillary data.

\section{A.1. Large Spitzer Separations}

There are eight ALMA sources in Figure 3 which have Spitzer YSO separations of $>14^{\prime \prime}$. One of these was presented in Section 3.4, source 37. A further three of the eight sources, source 10, 11, and 12, appear in Figure 5.

Figure 10 shows the remaining four ALMA sources, sources $5,19,23$, and 27, which lie in three separate fields. As can be seen in the figure, three of the four ALMA detections in Figure 10 appear to be separated from both the SCUBA core peak and a central Spitzer YSO. There are subtle indications for all three ALMA detections that they lie on local SCUBA-2 flux maxima which were not prominent enough to be identified as distinct cores in Jørgensen et al. (2008). ${ }^{14}$

\section{A.2. Moderate Spitzer Separations}

In Figures 11 and 12, we show the five ALMA detections which have moderate separations $\left(4^{\prime \prime}-7^{\prime \prime}\right)$ from the nearest Spitzer YSO, for which Herschel observations revealed protostellar counterparts.

\section{A.3. Small Spitzer Separations}

We have a large number of ALMA detections which are strongly associated (separations of $<2^{\prime \prime}$ ) with a Spitzer YSO. Figures 13 and 14 show single pointing fields, while Figures 15-17 show mosaicked areas.

As noted in Section 2, our initial sample contained 14, 4, and 5 SCUBA cores associated with Class $0+\mathrm{I}$, Flat, and Class II YSOs, respectively. Of these, we detected 24, 3, and 5 sources with ALMA across 13, 3, and 5 unique cores, respectively. In other words, only one Class $0+\mathrm{I}$ and one Flat core had no ALMA detections within the field of view. Since earlier classes of protostars generally have more surrounding dust than later classes, these two non-detections are surprising. One possibility is that these protostars are at a later stage of evolution, but are viewed edge-on, making them appear younger. As discussed earlier, some of the ALMA detections in the protostellar core fields are located a significant distance from the nearest Spitzer YSO location. Nearly every protostellar core, however, had at least one detection which was clearly associated with a Spitzer YSO. The lone exception is the Class II core 162659-24454, where ALMA detected a single emission peak located $\sim 6^{\prime \prime}$ from the nearest Spitzer YSO listed in Dunham et al. (2015). This source is shown in Figure 11. Examination of Herschel observations in this region suggest that the Spitzer catalog may have a positional offset, as Herschel emission from a YSO is centered on the ALMA detection. We defer an indepth examination of the properties of ALMA's protostellar detections to a future paper.

\footnotetext{
${ }^{14}$ We also checked the Pattle et al. (2015) SCUBA-2-based core catalog, bu did not find close positional associations between any of these detections and additional cores identified by Pattle et al. (2015).
} 

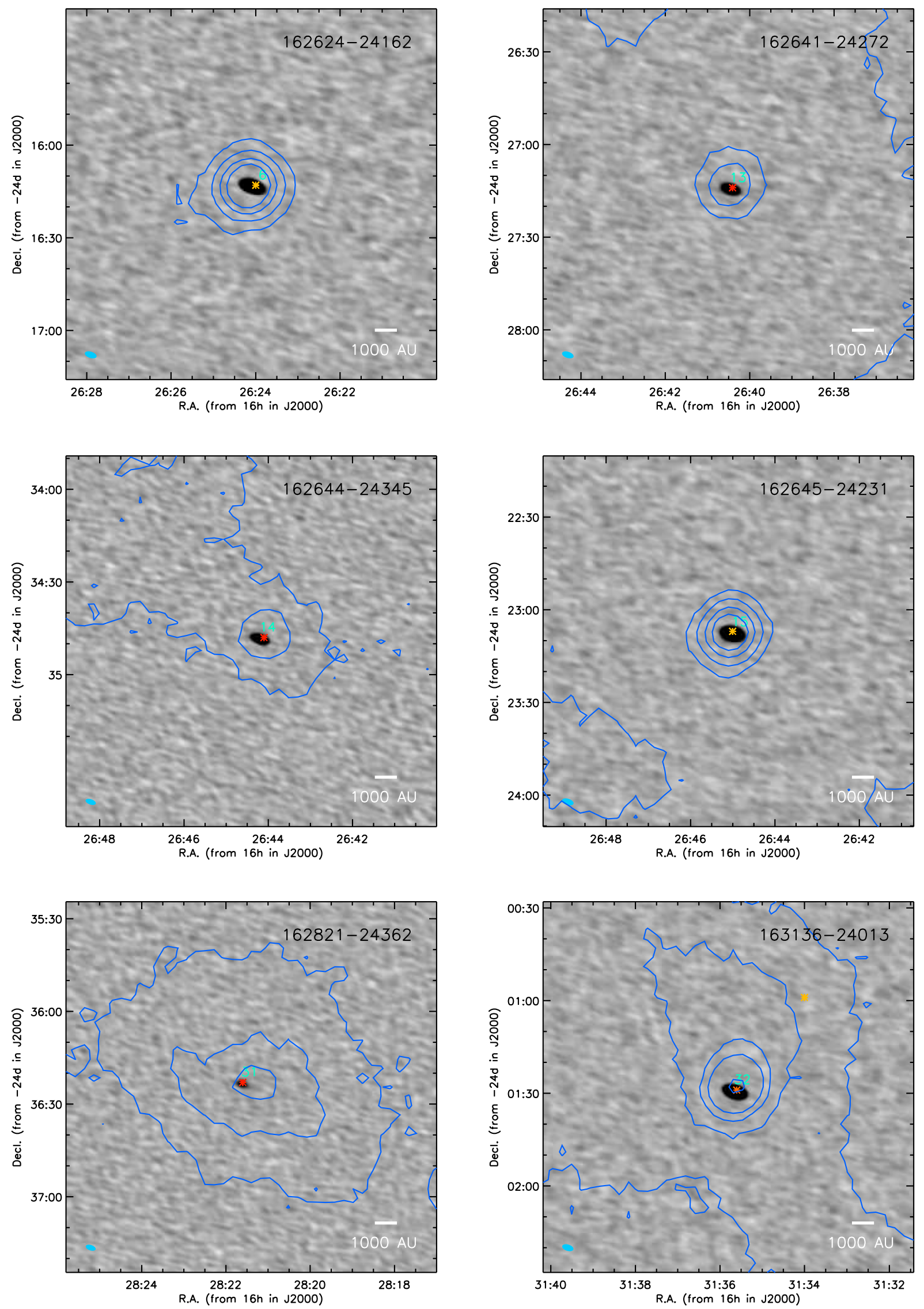

Figure 13. ALMA detections coincident with a Spitzer YSO for six single-pointing fields. See Figures 4 and 5 for the plotting conventions used. 

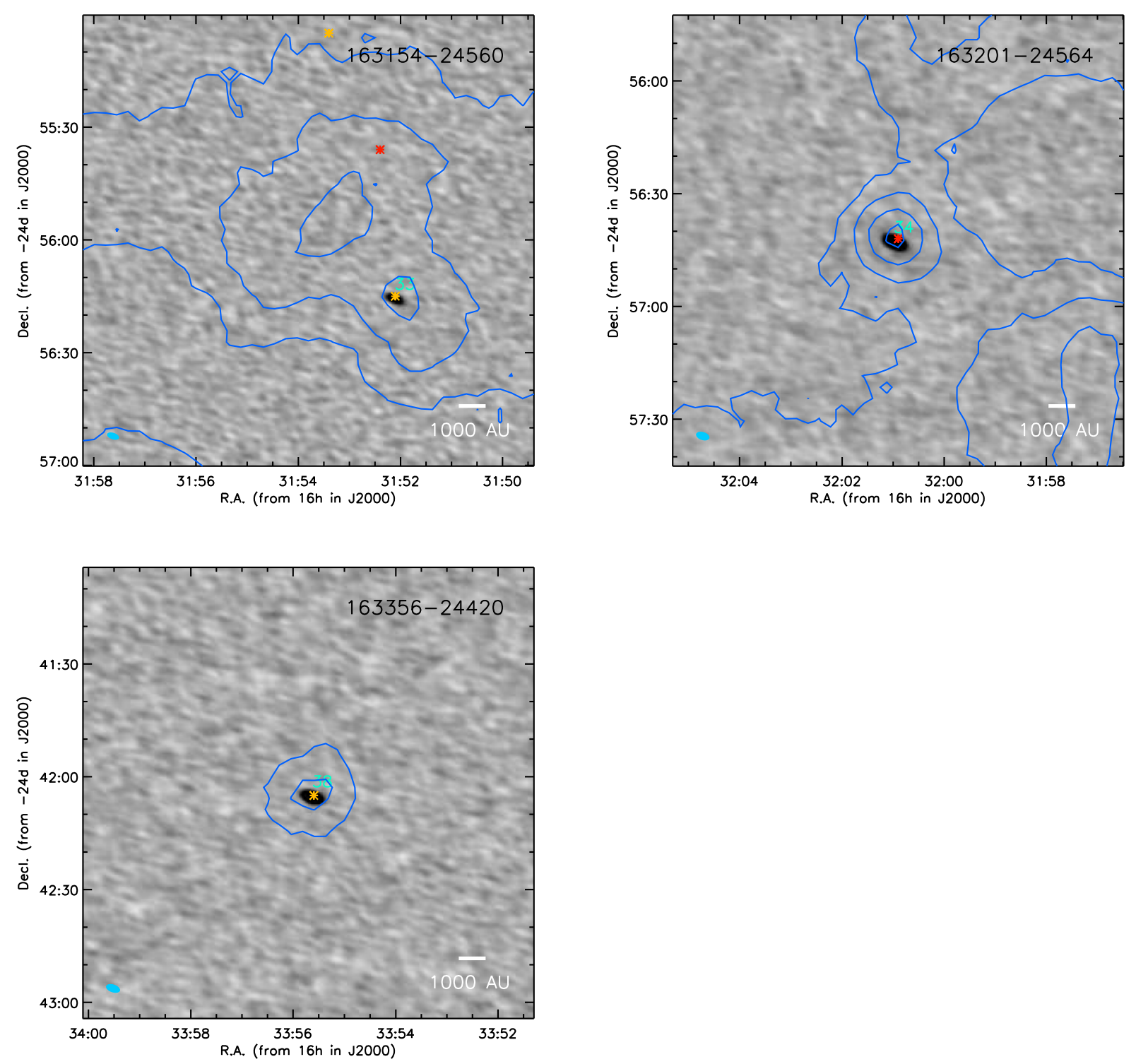

Figure 14. ALMA detections coincident with a Spitzer YSO for the remaining three single-pointing fields. See Figures 4 and 5 for the plotting conventions used.

\section{A.4. Protostellar Multiplicity}

The ALMA detections contain a number of pairs with projected separations of a few thousand au. These include previously identified wide protostellar binaries IRAS 162932422 (sources 35 and 36 in Figure 11) and VLA 1623 and VLA $1623 \mathrm{~W}$ (sources 8 and 9 in Figure 5). Altogether 14 of our 38 detections, or one third, have a second source with projected distance $\lesssim 3000$ au. Figure 5 contains a particularly interesting example which could represent a forming triple or a forming binary with an unbound single protostar. Extended filamentary emission appears to connect all sources in the area, also previously observed at larger angular scales by Motte et al. (1998) and Johnstone et al. (2000), for example, which increases the confidence that they are forming together within a single structure.

Pairs with separations of $\sim 1000-3000$ au may indicate the occurrence of turbulent fragmentation, which some numerical simulations predict may produce wide protobinaries on these size scales (Offner et al. 2010, 2016). Observations of a wideseparation forming bound quadruple system was recently presented by Pineda et al. (2015), where more detailed velocity information was available. In a VLA survey of Perseus, Tobin et al. (2016b) found more than half of identified protostellar pairs had projected separations greater than a few hundred au. They estimated the overall protostellar multiplicity fraction in Perseus is $0.40 \pm 0.06$. Thus, the fractions of potential widebinaries in our Ophiuchus sample and Perseus are roughly consistent. ${ }^{15}$

We caution, however, that projected proximity does not guarantee sources are gravitationally bound or will go on to form a binary star system. Pairs may have more significant separations along the line-of-sight or be chance alignments.

\footnotetext{
${ }^{15}$ Our ALMA observations are sensitive to multiple systems with separations greater than several hundred au, and show a similar distribution in the thousands of au range to the Tobin et al. (2016b) Perseus results.
} 


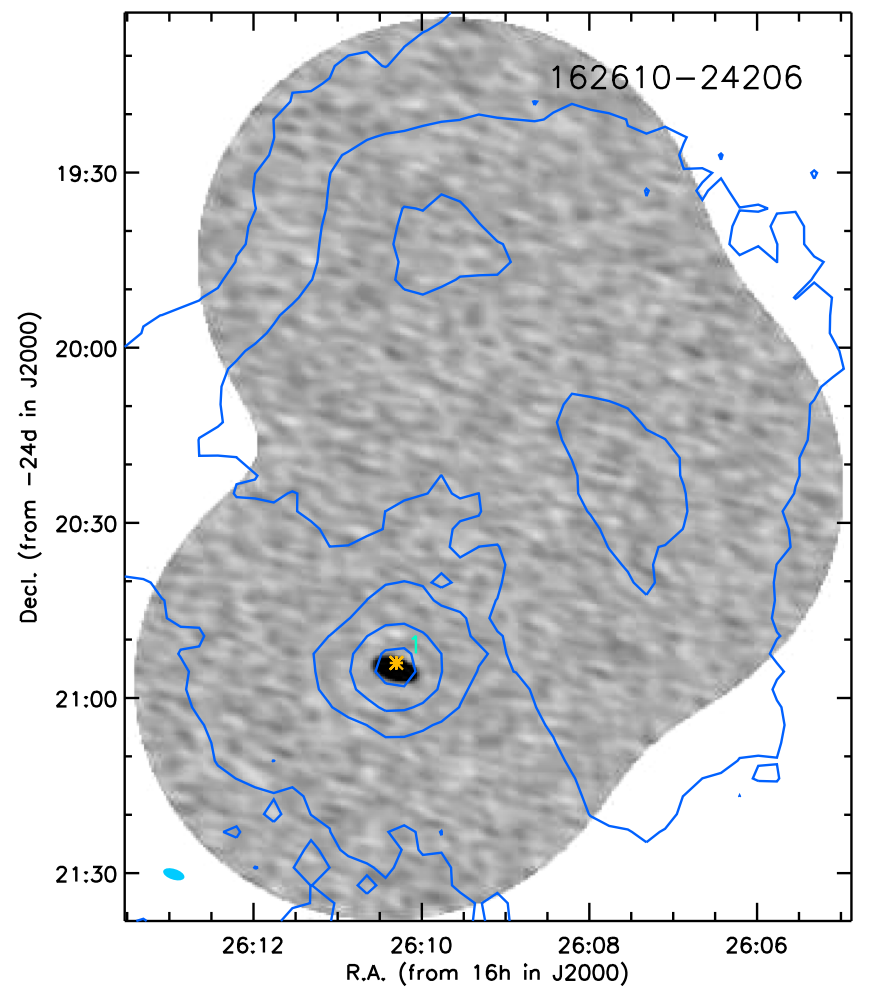

Figure 15. ALMA detection coincident with a Spitzer YSO in the mosaicked field 162610-24206. See Figures 4 and 5 for the plotting conventions used.

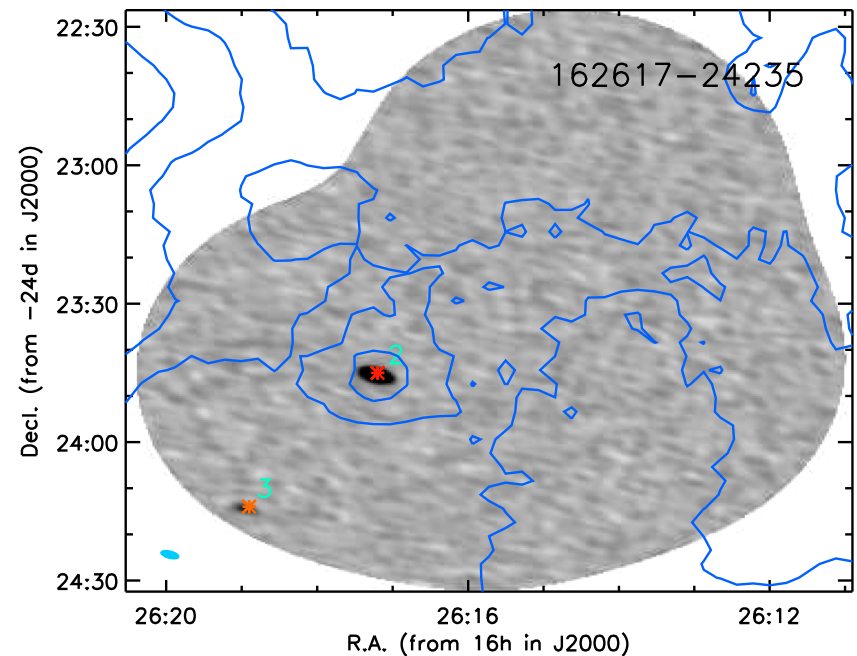

Figure 16. ALMA detections coincident with Spitzer YSOs in the mosaicked field 162617-24235. See Figures 4 and 5 for the plotting conventions used.

In addition, significant dynamical evolution, which impacts the separation distribution, is expected to occur during the protostellar phase (e.g., Bate et al. 2002; Tobin et al. 2016b). Sources with initially wide separations may migrate to shorter separations on $\sim 0.1 \mathrm{Myr}$ timescales or become unbound (Offner et al. 2010, 2016). Velocity information is required to draw conclusions about the boundedness of close pairs in our sample and to determine whether such pairs are likely to remain bound (e.g., Pineda et al. 2015).

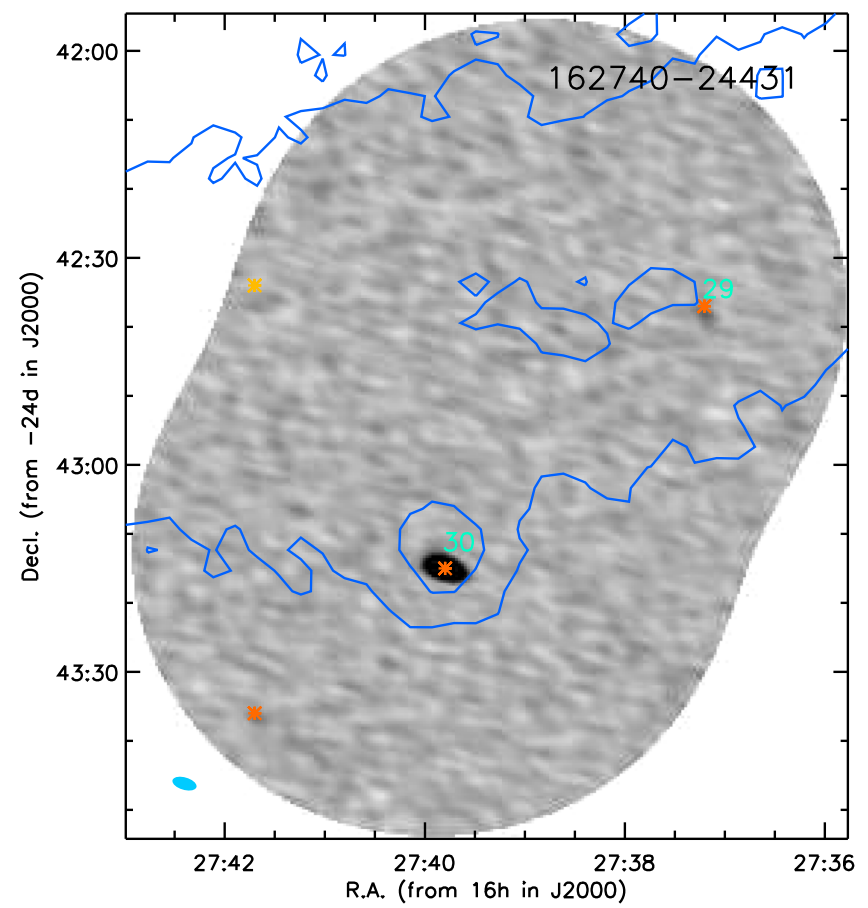

Figure 17. ALMA detections coincident with Spitzer YSOs in the mosaicked field 162740-24431. See Figures 4 and 5 for the plotting conventions used.

\section{References}

André, P., Men'shchikov, A., Bontemps, S., et al. 2010, A\&A, 518, L102 Bate, M. R., Bonnell, I. A., \& Bromm, V. 2002, MNRAS, 336, 705 Belloche, A., Schuller, F., Parise, B., et al. 2011, A\&A, 527, A145 Bonnor, W. B. 1956, MNRAS, 116, 351

Bourke, T. L., Myers, P. C., Caselli, P., et al. 2012, ApJ, 745, 117

Chen, X., \& Arce, H. G. 2010, ApJL, 720, L169

Chen, X., Arce, H. G., Zhang, Q., et al. 2013, ApJ, 768, 110

Connelley, M. S., Reipurth, B., \& Tokunaga, A. T. 2008, AJ, 135, 2526

Di Francesco, J., Evans, N. J., II, Caselli, P., et al. 2007, in Protostars and Planets V, ed. B. Reipurth, D. Jewitt, \& K. Keil (Tucson, AZ: Univ. Arizona Press), 17

Duchêne, G., \& Kraus, A. 2013, ARA\&A, 51, 269

Dunham, M. M., Allen, L. E., Evans, N. J., II, et al. 2015, ApJS, 220, 11

Dunham, M. M., Crapsi, A., Evans, N. J., II, et al. 2008, ApJS, 179, 249

Dunham, M. M., Offner, S. S. R., Pineda, J. E., et al. 2016, ApJ, 823, 160

Duquennoy, A., \& Mayor, M. 1991, A\&A, 248, 485

Ebert, R. 1955, ZA, 37, 217

Enoch, M. L., Evans, N. J., II, Sargent, A. I., et al. 2008, ApJ, 684, 1240

Evans, N. J., II, Dunham, M. M., Jørgensen, J. K., et al. 2009, ApJS, 181, 321

Fisher, R. T. 2004, ApJ, 600, 769

Friesen, R. K., Di Francesco, J., Bourke, T. L., et al. 2014, ApJ, 797, 27

Friesen, R. K., Di Francesco, J., Shirley, Y. L., \& Myers, P. C. 2009, ApJ, 697, 1457

Gagné, M., Skinner, S. L., \& Daniel, K. J. 2004, ApJ, 613, 393

Goodwin, S. P., Nutter, D., Kroupa, P., Ward-Thompson, D., \& Whitworth, A. P. 2008, A\&A, 477, 823

Goodwin, S. P., Whitworth, A. P., \& Ward-Thompson, D. 2004, A\&A, 423,169

Holman, K., Walch, S. K., Goodwin, S. P., \& Whitworth, A. P. 2013, MNRAS, 432,3534

Jessop, N. E., \& Ward-Thompson, D. 2000, MNRAS, 311, 63

Johnstone, D., Di Francesco, J., \& Kirk, H. 2004, ApJL, 611, L45

Johnstone, D., Wilson, C. D., Moriarty-Schieven, G., et al. 2000, ApJ, 545, 327

Jørgensen, J. K., Johnstone, D., Kirk, H., et al. 2008, ApJ, 683, 822

Jørgensen, J. K., Johnstone, D., Kirk, H., \& Myers, P. C. 2007, ApJ, 656, 293

Kainulainen, J., Stutz, A. M., Stanke, T., et al. 2016, arXiv:1603.05688

Kirk, J. M., Crutcher, R. M., \& Ward-Thompson, D. 2009, ApJ, 701, 1044

Kratter, K., \& Lodato, G. 2016, ARA\&A, 54, 271 
Lee, K., Looney, L. W., Schnee, S., \& Li, Z.-Y. 2013, ApJ, 772, 100

Li, P. S., Martin, D. F., Klein, R. I., \& McKee, C. F. 2012, ApJ, 745, 139

Lin, D., Webb, N. A., \& Barret, D. 2012, ApJ, 756, 27

Lis, D. C., Wootten, H. A., Gerin, M., et al. 2016, ApJ, 827, 133

Lomax, O., Whitworth, A. P., Hubber, D. A., Stamatellos, D., \& Walch, S 2015, MNRAS, 447, 1550

Looney, L. W., Mundy, L. G., \& Welch, W. J. 2000, ApJ, 529, 477

Mairs, S., Johnstone, D., Offner, S. S. R., \& Schnee, S. 2014, ApJ, 783, 60

Molinari, S., Schisano, E., Faustini, F., et al. 2011, A\&A, 530, A133

Motte, F., Andre, P., \& Neri, R. 1998, A\&A, 336, 150

Nakamura, F., Takakuwa, S., \& Kawabe, R. 2012, ApJL, 758, L25

Nutter, D., Ward-Thompson, D., \& André, P. 2006, MNRAS, 368, 1833

Offner, S. S. R., Capodilupo, J., Schnee, S., \& Goodman, A. A. 2012, MNRAS, 420, L53

Offner, S. S. R., Dunham, M. M., Lee, K. I., Arce, H. G., \& Fielding, D. B. 2016, ApJL, 827, L11

Offner, S. S. R., Kratter, K. M., Matzner, C. D., Krumholz, M. R., \& Klein, R. I. 2010, ApJ, 725, 1485

Ortiz-León, G. N., Loinard, L., Kounkel, M. A., et al. 2017, ApJ, 834, 141

Ossenkopf, V., \& Henning, T. 1994, A\&A, 291, 943

Padgett, D. L., Rebull, L. M., Stapelfeldt, K. R., et al. 2008, ApJ, 672, 1013
Pattle, K., Ward-Thompson, D., Kirk, J. M., et al. 2015, MNRAS, 450, 1094 Pineda, J. E., Goodman, A. A., Arce, H. G., et al. 2011, ApJL, 739, L2

Pineda, J. E., Offner, S. S. R., Parker, R. J., et al. 2015, Natur, 518, 213

Pineda, J. E., Rosolowsky, E. W., \& Goodman, A. A. 2009, ApJL, 699, L134

Raghavan, D., McAlister, H. A., Henry, T. J., et al. 2010, ApJS, 190,

Sadavoy, S. I., Di Francesco, J., Bontemps, S., et al. 2010a, ApJ, 710, 1247

Sadavoy, S. I., Di Francesco, J., \& Johnstone, D. 2010b, ApJL, 718, L32

Schnee, S., Di Francesco, J., Enoch, M., et al. 2012, ApJ, 745, 18

Schnee, S., Enoch, M., Johnstone, D., et al. 2010, ApJ, 718, 306

Shinnaga, H., Humphreys, E., Indebetouw, R., et al. 2015, in ASP Conf. Ser. 499, Revolution in Astronomy with ALMA: The Third Year, ed. D. Iono et al. (San Francisco, CA: ASP), 355

Stanke, T., Smith, M. D., Gredel, R., \& Khanzadyan, T. 2006, A\&A, 447, 609 Stutz, A. M., Tobin, J. J., Stanke, T., et al. 2013, ApJ, 767, 36

Tobin, J. J., Kratter, K. M., Persson, M. V., et al. 2016a, Natur, 538, 483

Tobin, J. J., Looney, L. W., Li, Z.-Y., et al. 2016b, ApJ, 818, 73

Wenger, M., Ochsenbein, F., Egret, D., et al. 2000, A\&AS, 143, 9

Williams, J. P., de Geus, E. J., \& Blitz, L. 1994, ApJ, 428, 693

Young, K. E., Enoch, M. L., Evans, N. J., II, et al. 2006, ApJ, 644, 326

Zhang, M., Brandner, W., Wang, H., et al. 2013, A\&A, 553, A41 